\title{
Large-Eddy Simulation, Fuel Rod Vibration and Grid-to-Rod Fretting in Pressurized Water Reactors
}

\author{
Mark A. Christon ${ }^{\mathrm{a}, \mathrm{b}}$, Roger Lu ${ }^{\mathrm{d}}$, Jozsef Bakosi ${ }^{\mathrm{c}}$, Balasubramanya T. \\ Nadiga $^{c}$, Zeses Karoutas ${ }^{\mathrm{d}}$, Markus Berndt ${ }^{\mathrm{c}, *}$ \\ ${ }^{a}$ Computational Sciences International, \\ Los Alamos, NM 87544, USA \\ ${ }^{b}$ Guest Scientist - Computational Physics and Methods Group (CCS-2) \\ Los Alamos National Laboratory, Los Alamos, NM 87545, USA \\ ${ }^{c}$ Computational Physics and Methods Group (CCS-2), \\ Los Alamos National Laboratory, Los Alamos, NM 87545, USA \\ ${ }^{d}$ Westinghouse Electric Company, \\ Hopkins, SC 29061 USA
}

\begin{abstract}
Grid-to-rod fretting (GTRF) in pressurized water reactors is a flow-induced vibration phenomenon that results in wear and fretting of the cladding material on fuel rods. GTRF is responsible for over $70 \%$ of the fuel failures in pressurized water reactors in the United States. Predicting the GTRF wear and concomitant interval between failures is important because of the large costs associated with reactor shutdown and replacement of fuel rod assemblies. The GTRF-induced wear process involves turbulent flow, mechanical vibration, tribology, and time-varying irradiated material properties in complex fuel assembly geometries. This paper presents a new approach for predicting GTRF induced fuel rod wear that uses high-resolution implicit large-eddy simulation to drive nonlinear transient dynamics computations. The GTRF fluid-structure problem is separated into the simulation of the turbulent flow field in the complex-geometry fuel-rod bundles using implicit large-eddy simulation, the calculation of statistics of the resulting fluctuat-

\footnotetext{
*Corresponding author.

Email addresses: machriston@c-sciences.com (Mark A. Christon), lur@westinghouse.com (Roger Lu), jbakosi@lanl.gov (Jozsef Bakosi), balu@lanl.gov (Balasubramanya T. Nadiga), karoutze@westinghouse.com (Zeses Karoutas), berndt@lanl.gov (Markus Berndt)
}

Preprint submitted to Journal of Computational Physics

June 16, 2016

(C) 2016. This manuscript version is made available under the Elsevier user license http://www.elsevier.com/open-access/userlicense/1.0/ 
ing structural forces, and the nonlinear transient dynamics analysis of the fuel rod. Ultimately, the methods developed here, can be used, in conjunction with operational management, to improve reactor core designs in which fuel rod failures are minimized or potentially eliminated. Robustness of the behavior of both the structural forces computed from the turbulent flow simulations and the results from the transient dynamics analyses highlight the progress made towards achieving a predictive simulation capability for the GTRF problem.

Keywords: thermal-hydraulics, nuclear reactor, rod-bundles, grid-to-rod fretting, implicit large-eddy simulation, nonlinear structural dynamics, incompressible flow, monotonicity-preserving advection

\section{Introduction}

Grid-to-Rod Fretting (GTRF) is a complex flow-induced vibration phenomenon that causes cladding wear in the fuel rod assemblies of Pressurized Water Reactors (PWRs). Fuel rod wear due to GTRF is currently one of the primary causes of fuel failure. It is responsible for over $70 \%$ of the fuel leaks in PWRs in the United States, and costs power utilities millions of dollars in preventative measures. Understanding and predicting the GTRF wear is fundamental to estimating the interval between fuel assembly failures.

In the fuel assembly of a pressurized water reactor, shown in Figure 1, fuel rods are separated by spacer grids that have small preloaded retention springs that contact the rods. In order to augment the heat transfer, mixing vanes are appended to the spacer grids. As the coolant flows through the fuel assembly in a primarily axial direction, fluid forces generated by the turbulent flow induce fuel rod vibration resulting in fretting wear. As radiation induced creep and growth effects take place, the preloads in the retention springs relax and the so-called cladding "creep-down" and spacer grid growth occurs. Under these conditions, the springs, that secure the fuel rod, may lose contact with the fuel rod, forming a gap between the spacer grid and the fuel rod. This magnifies the effects of flow-induced vibration and causes both normal and tangential cyclic contact forces to be generated between the retention springs and the fuel rods, further aggravating the fretting wear. In addition, the changes in the mechanical behavior and wear characteristics of the materials in the radiation environment, present during reactor operation, have a direct effect on the GTRF mechanisms. To date, it has not been pos- 
sible to completely characterize the flow-induced Fluid-Structure Interaction (FSI) problem for GTRF. Indeed, given the turbulent nature of the coolant flow, the relatively high Reynolds number, and the flexible character of the fuel rods, the FSI problem at the reactor core scale is daunting.
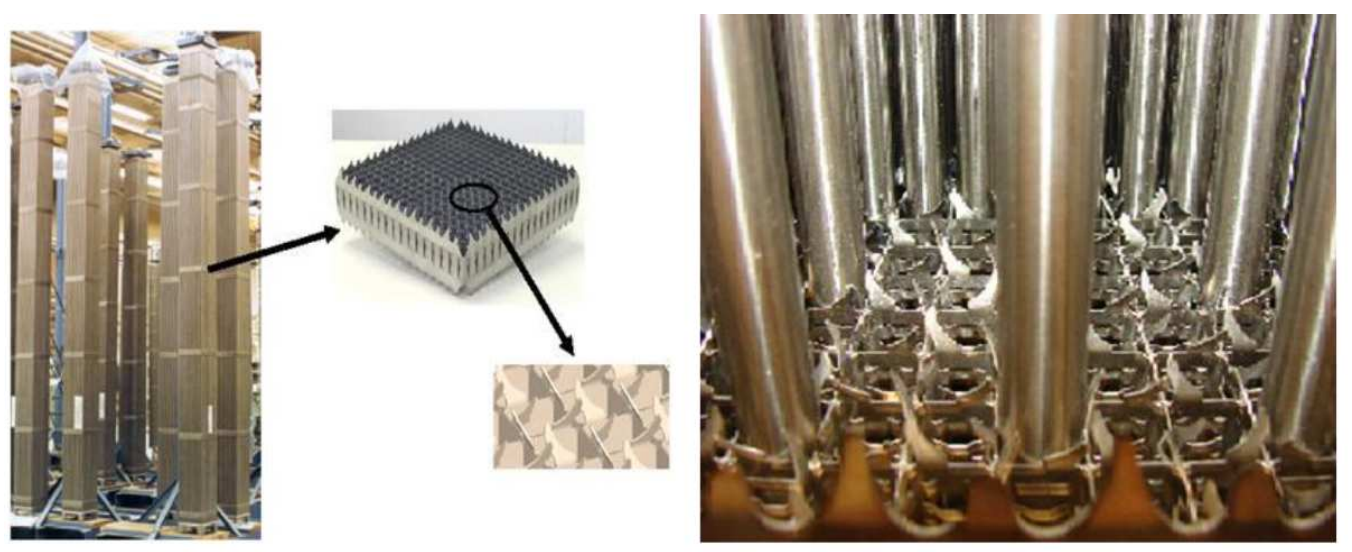

Figure 1: PWR fuel rod assembly, rod spacer grid and mixing vanes. Reproduced without permission from Liu [1].

There are a number of flow-induced vibration problems in a nuclear power plant that involve the reactor, associated piping, heat exchangers, steam generators, and ancillary diagnostic equipment [2]. Pettigrew, et al. [3] consider a broad array of flow-induced vibration problems, albeit specialized to the CANDU reactor configuration. We note that the CANDU reactor can experience wear at the appendages of the outer ring of the fuel rod bundle, but this is not to be confused with GTRF. A complete review of the work associated with all possible flow-induced reactor vibration problems is beyond the scope of this work. However, a brief review of the work related to the application of Computational Fluid Dynamics (CFD) for flow-induced vibration problems in reactor cores and fuel assemblies is presented.

Ikeno and Kajishima [4] applied Large-Eddy Simulation (LES) to the flow downstream of mixing vanes in a rod bundle. They used an immersedboundary technique to treat the complex geometry and a dynamic subgridscale turbulence model to examine the mixing grid wake and downstream swirl. Benhamadouce, et al. [5] performed an LES of the flow in the subchannels surrounding a single rod, and subsequently used the turbulent forces to compute the elastic vibration of the fuel rod. Here, a relatively coarse mesh 
with 8 million cells was used for the $R e=30,000$ flow. Related work by Kim [6-8] has considered GTRF wear models for PWRs as well as the effects of the rod support conditions on fuel rod vibration.

Conner, et al. [9] present a validation study using a $5 \times 5$ rod bundle, representative of a fuel assembly and compare the mixing-vane-induced swirl with Particle Image Velocimetry (PIV) data. Here, the RNG $k-\epsilon$ model was used with a wall resolution corresponding to $40 \leq y^{+} \leq 100$ to compute a steady-state solution. Yan, et al. [10] performed time-accurate CFD computations and compared the effect of the so-called "protective grid" at the fuel-inlet region of a reactor. For this study, meshes with 7, 16, and 60 million cells were used. Here, it was shown that a time-accurate CFD calculation can be used to determine transient fuel rod forces for subsequent dynamic analyses. This work also demonstrated that the protective grid significantly reduces flow-induced vibration at the reactor core inlet. Zhang and Yu [11], and Bhattacharya, et al. [12] have performed LES of flow in CANDU fuel bundles and observed the vortex shedding phenomena associated with the end plates. The work by Delafontaine and Ricciardi [13] used LES to determine the time-dependent rod forces in a $3 \times 3 \mathrm{PWR}$ rod bundle. Here detailed information about the angular variation of pressure forces on the fuel rod are presented. The work by Liu, et al. [14] considered fluid-structure interaction in simplified fuel assemblies where a rod buckling instability was demonstrated to occur with large axial flow velocities. Mohany and Hassan [15] modeled the flow-induced vibration and associated pressure tube fretting in a CANDU fuel bundle.

This paper presents a rigorous approach for predicting GTRF induced fuel rod wear using high-resolution Implicit Large-Eddy Simulation (ILES) coupled to nonlinear transient structural dynamics. The GTRF problem is separated into sequential steps that involve: a) simulation of the turbulent flow field in the complex-geometry fuel-rod bundles, b) calculation of the resulting statistics of the fluctuating structural forces, and c) the nonlinear transient dynamics of the fuel rod using the computed structural loads. The ILES are performed using Hydra-TH which is built on the Hydra Toolkit, a $\mathrm{C}++$ multiphysics toolkit for the rapid development of parallel scalable simulation tools. The methodology presented here may ultimately be used to improve reactor core designs so that fuel failures are minimized or potentially eliminated.

The ILES procedure is first validated using a $5 \times 5$ rod bundle configuration and comparing simulated and experimentally-measured velocities im- 
mediately downstream of the mixing vanes. Next, highly-instrumented ILES of the $3 \times 3$ rod bundle flow using a sequence of four meshes with increasing resolution are discussed. The computed fuel rod forces and associated statistics downstream of the mixing vanes are seen to fall in an asymptotic regime at the finer three resolutions, and this feature of the numerics is exploited to obtain the limiting values of the fuel rod forces using Richardson extrapolation. Furthermore, uncertainty in the fuel rod forces due to turbulent intermittency are estimated using time-series data from the one second turbulent computations. The Root-Mean Squared (RMS) fluid forces on the fuel rod from the ILES and Richardson extrapolation are used as input forces to VITRAN (VIbration TRansient Analysis - Nonlinear), a proprietary code developed by Westinghouse. VITRAN is used to perform nonlinear structural dynamics and wear work-rate calculations.

The rest of the paper is organized as follows. The methods used to compute the fluid flow, the loads on the fuel rods, and the nonlinear structural dynamics are briefly outlined in $\S 2$. A validation study using a $5 \times 5$ rod bundle configuration, is presented in $\S 3$ and followed by $3 \times 3$ calculations and GTRF wear studies. Finally, summary, conclusions, and future directions are presented in $\S 4$.

\section{Problem Formulation}

The methods used for the ILES and transient structural dynamics are outlined in $\S 2.1$, and $\S 2.2$, respectively.

\subsection{Implicit Large-Eddy Simulation}

Light water is the primary coolant in PWRs, and at the operating pressures and temperatures may be treated as an incompressible flow. The Navier-Stokes equations for an incompressible fluid are

$$
\begin{gathered}
\nabla \cdot \mathbf{v}=0 \\
\frac{\partial \rho \mathbf{v}}{\partial t}+\nabla \cdot(\rho \mathbf{v} \mathbf{v})=\nabla \cdot(-p \mathbf{I}+\boldsymbol{\tau})+\rho \mathbf{f}
\end{gathered}
$$

defined in the domain $\Omega$ with boundary $\Gamma$ in the time interval $[0, T]$. Here, $\rho$ is the mass density, $\mathbf{v}=\left(v_{x}, v_{y}, v_{z}\right)^{T}$ is the velocity, $p$ is the fluid pressure, $\boldsymbol{\tau}$ is the deviatoric stress, and $\mathbf{f}$ is the body force per unit mass. A constitutive equation relates the deviatoric stress and the strain rate, $\boldsymbol{\tau}=2 \mu \mathbf{S}$, where $\mu$ is the molecular viscosity and $\mathbf{S}=\frac{1}{2}\left[\nabla \mathbf{v}+(\nabla \mathbf{v})^{T}\right]$. 
The momentum equations, Eq. (2), are subject to boundary conditions that consist of prescribed velocity $\mathbf{v}(\mathbf{x}, t)=\hat{\mathbf{v}}(\mathbf{x}, t)$ on $\Gamma_{1}$, or prescribed traction $\{-p \mathbf{I}+2 \mu \mathbf{S}\} \cdot \mathbf{n}=\hat{\mathbf{f}}(\mathbf{x}, t)$ on $\Gamma_{2}$ where $\Gamma=\Gamma_{1} \cup \Gamma_{2}$ is the domain boundary with outward normal $\mathbf{n}$. A detailed discussion of boundary conditions for the incompressible Navier-Stokes equations may be found in [16].

Velocity initial conditions are prescribed as $\mathbf{v}(\mathbf{x}, 0)=\mathbf{v}^{0}(\mathbf{x})$. For a mathematically well-posed incompressible flow problem, the prescribed initial and boundary conditions on the velocity field must satisfy

$$
\begin{gathered}
\nabla \cdot \mathbf{v}^{0}=0 \\
\mathbf{n} \cdot \mathbf{v}(\mathbf{x}, 0)=\mathbf{n} \cdot \mathbf{v}^{o}(\mathbf{x})
\end{gathered}
$$

If $\Gamma_{2}=0$, i.e., enclosure flows with $\mathbf{n} \cdot \mathbf{v}$ prescribed on all surfaces, then

$$
\int_{\Gamma} \mathbf{n} \cdot \mathbf{v}^{0} d \Gamma=0
$$

must also be satisfied.

For GTRF, the incompressible flow solver in the Hydra Toolkit, "HydraTH", uses a high-resolution monotonicity-preserving finite volume discretization derived in the framework of discontinuous-Galerkin methods. The solution algorithm relies on a second-order projection method using a co-velocity technique to guarantee a divergence-free advective velocity at each time step. A finite-volume method with a monotonicity-preserving advection method is used for all transported variables, and a finite-element method is used for the pressure. For time-accurate problems, a second-order accurate trapezoidal method is used with an implicit treatment of advective terms. The time-integrator is neutrally-dissipative, and in conjunction with the projection method, Hydra-TH provides a robust and accurate ILES capability. As pointed out by Grinstein, et al. [17, pg. 197], "Thus, all algorithms based on finite-volume differencing have a truncation term in the form of the divergence of a subgrid-scale model." From this perspective, the ILES capabilities in Hydra-TH derive from the high-resolution monotonicity-preserving advective treatment as described in Christon, et al. [18].

From a practical point of view, the most useful information that can be obtained from simulations of turbulent flows are statistical and integrated quantities derived from the fluctuating flow fields. Examples of statistics are the mean velocity, the RMS pressure, and the turbulent kinetic energy spectrum. Examples of integrated quantities are the mean drag or mean lift 
on an airplane wing or the Power Spectral Density (PSD) distribution of the fluctuating forces that load the fuel rods in a pressurized water nuclear reactor.

The typical turbulent flow representation using the Reynolds-Averaged Navier-Stokes (RANS) equations augmented by a turbulence model, directly compute the flow statistics by solving for only the mean flow field values, and depending on the model, the second moments. For example, the $k$ - $\epsilon$ model is a popular way to approximate the effects of fluctuations on the mean velocity, see for example Pope [19]. It is important to understand that the main goal of the $k$ - $\epsilon$ model is to provide closure for the mean velocity via the time scale $\sim k / \epsilon$. Consequently, one can expect a statistically meaningful description of the mean but less of the fluctuations, e.g., $k$ or $\epsilon$. If the second moments, e.g., the fluctuations about the mean, are also important, a model with a higher level of statistical approximation is required.

In comparison to RANS, LES has the advantage of describing unsteady, large-scale turbulent structures, and hence can be used to study phenomena such as the unsteady loads that drive the GTRF problem. In LES, the small dynamical scales are modeled, while the large unsteady eddies are grid-resolved. If the small scales are universal, i.e., Kolmogorov's hypothesis holds, as in many engineering applications where the turbulent kinetic energy is predominantly generated by shear production, LES is known to provide excellent results, both because a larger fraction of the energetically important features are explicitly represented as compared to RANS and because the effect of the small, modeled scales on the large scales are smaller than in RANS. We believe a problem such as GTRF can effectively be studied with ILES.

Remark 1. There are some fundamental conceptual issues in applying RANS models in a time-dependent sense to calculate RMS forces for GTRF. As shown in Figure 2, RANS relies on an implied time-scale, to separate the high-frequency oscillations from the longer time-scale variation in the computed mean variables, e.g., velocity and pressure. When flows are slowly varying, and the long-period variations associated with a time-period $T$ can be separated from the time-scale for the fluctuations, then time-dependent unsteady RANS may be appropriate. For GTRF applications, there is not a clear separation of time-scales, and in fact, the resolved time-scale $T$ approaches the averaging time-scale making unsteady RANS difficult to interpret, understand and apply in a reliable way when computing the RMS forces 
required for GTRF analyses. Future efforts may investigate alternative analysis techniques that would permit a clear separation of time-scales and admit the use of unsteady RANS.

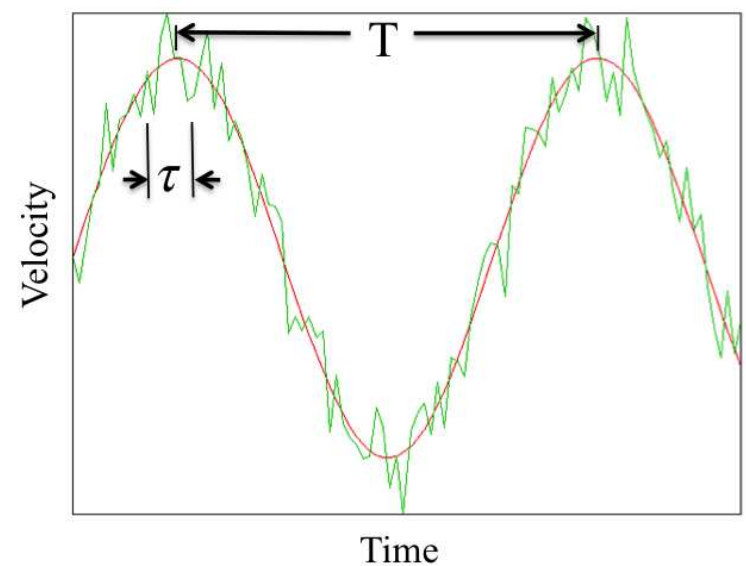

Figure 2: Time-averaging for unsteady RANS showing the instantaneous velocity in green and the time-averaged velocity in red.

\section{Assessment of Turbulence Models}

In order to support our assertion that LES, specifically ILES, can be used to effectively compute the RMS forces for GTRF, we briefly summarize a comparison of three different turbulence models with a mesh refinement study using 5 meshes. The three turbulence models used were: (a) ILES, (b) Detached-Eddy Simulation (DES), a blended LES/RANS method, and (c) the Spalart-Allmaras RANS model. ILES is inherently time-accurate, and requires that the large-eddies be spatially resolved. The DES model is a blended model that switches between an LES model, in regions where the spatial resolution is sufficiently fine, and the Spalart-Allmaras model. Typically, in regions near solid boundaries, the model switches to SpalartAllmaras making use of its wall-damping functions. The Spalart-Allmaras model is a RANS model that is exercised in a time-accurate mode. Addition details of this comparison may be found in $\mathrm{Lu}$, et al. [20], and the discrete formulation of the models is detailed in Christon [21]. 
A set of 5 grids were used to test the sensitivity of the fuel rod forces to the dynamical approximations inherent in each turbulence model. All calculations in the model assessment were performed in a time-accurate way using the neutrally dissipative second-order trapezoidal rule time-integrator for 1.0 second of physical time. Note that a statistically stationary state was achieved at $t \approx 0.2$ seconds in all computations. A fixed $C F L$ condition, i.e., $C F L=4$, was used for all calculations in conjunction with an implicit second-order treatment of the advective and diffusive terms. The flow-induced forces are computed on the fuel rod surface using

$$
\mathbf{F}(t)=-\int_{\Gamma} p(t) \mathbf{n} d \Gamma+2 \int_{\Gamma} \mu \mathbf{S} \cdot \mathbf{n} d \Gamma
$$

Figure 3 shows time-history plots of the components of the fuel-rod forces for the three turbulence models. All three models yield statistically stationary forces for $t \geq 0.2$ seconds. Although all three models yield some level of fluctuating forces on the rod surface, it is clear that the amplitude computed for DES and Spalart-Allmaras is damped significantly relative to the ILES results. Based on the time-history data, the RMS forces are somewhat muted as well, with the DES amplitude being reduced by approximately 15 to $17 \%$ relative to the ILES forces. Thus, we have adopted ILES for subsequent GTRF analyses.

\section{ILES Analysis Workflow}

Typically our ILES workflow begins with a preliminary grid that provides a wall-resolution with $1 \leq y^{+} \leq 10$ on average, where $y^{+}=u_{\tau} y / \nu$ is the nondimensional wall-normal distance, $u_{\tau}$ is the friction velocity, and $\nu$ is the fluid kinematic viscosity. By performing a short calculation in Hydra- $\mathrm{TH}$, the computed $y^{+}$values are calculated, and a mesh assessment following the procedure outlined by Bakosi, et al. [22] is performed. Once a the wall resolution is established, a preliminary LES is performed, and time-history data is collected and assessed to determine when a stationary flow is established. Our metrics for a statistically stationary flow depend on the domain-integrated kinetic energy $\left(\mathrm{KE}=\frac{1}{2} \int_{\Omega} \rho \mathbf{v} \cdot \mathbf{v} \mathrm{d} \Omega\right)$, as well as physical parameters such as the velocity, pressure, and surface forces. After a time-scale is identified for a statistically-stationary flow, the computation is repeated and a series of windowed time-averages are computed with derived statistics, e.g., means, RMS values. The sensitivity of the windowed averages is tested with increasing window size until a small error in derived statistics is determined. Once 


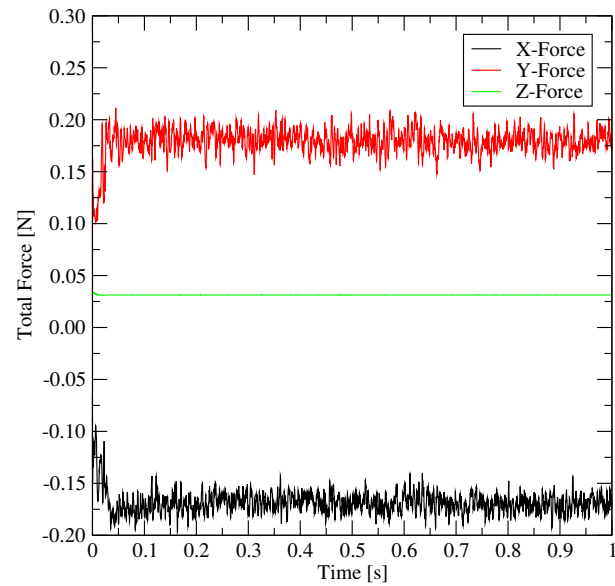

(a) ILES

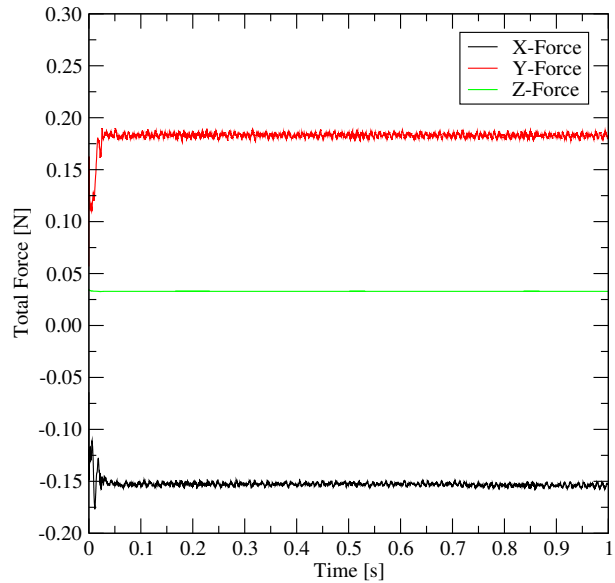

(b) DES

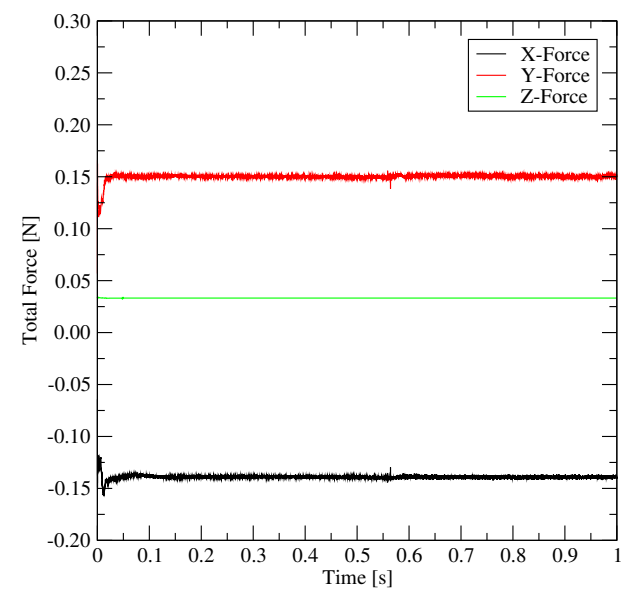

(c) Spalart-Allmaras

Figure 3: Instantaneous forces on the fuel rod for (a) ILES, (b) DES and (c) SpalartAllmaras turbulence models The Z-Force is the streamwise shear force on the fuel rod surface, and the $\mathrm{X}$ - and Y-Force are transverse to the flow direction. 
these preliminary computations are completed, we proceed with further mesh sensitivity studies and fully-instrumented ILES runs.

\subsection{Nonlinear Structural Dynamics}

VITRAN (Vibration Transient Analysis Non-linear) is a code developed by Westinghouse [23, 24], and is used to simulate vibration of nuclear fuel rods and the dynamic interaction between the fuel rods and their supports. The code also integrates an analysis method to predict GTRF performance, and includes hydraulic, structural, and tribological effects. VITRAN also provides statistical analysis modules with Monte Carlo capabilities that are particularly useful for including the large uncertainties existing in the mechanical parameters of a PWR fuel assembly and the associated boundary conditions.

In VITRAN, the dynamic response of a nuclear fuel rod and its supports is simulated using a nonlinear vibration model. Figure 4 shows a typical VITRAN model for a full-length fuel rod. The VITRAN formulation uses classic approximations and techniques, e.g., Euler-Bernoulli beam theory, and solves the following simplified equation for the rod motion

$$
\rho(y) \frac{\partial^{2} \mathbf{d}(y, t)}{\partial t^{2}}+c \frac{\partial \mathbf{d}(y, t)}{\partial t}+\frac{\partial^{2}}{\partial y^{2}}\left[E \cdot I \frac{\partial^{2} \mathbf{d}(y, t)}{\partial y^{2}}\right]=\mathbf{f}_{e}(y, t)+\mathbf{f}_{s}(y, t) .
$$

Here, $y$ is the coordinate direction aligned with the fuel rod axis and the streamwise direction of the flow. The displacement vector, $\mathbf{d}(y, t)=(u, w)^{T}$, is a two-dimensional vector of displacements in the $x$ - and $z$-coordinate directions that varies in $y, c$ is the viscous damping coefficient, $\rho(y)$ is the mass density per unit rod length, $E \cdot I$ is the bending stiffness, $\mathbf{f}_{e}(y, t)$ are the external excitation forces, and $\mathbf{f}_{s}(y, t)$ are the rod support forces (and are also two-dimensional). Additional details are provided by Lu, et al. [24].

The rod linear mass density $\rho(y)$ is determined from the fuel pellet and cladding masses, and it can also include the added hydraulic mass of the water surrounding the fuel rod. The rod stiffness is calculated from the clad stiffness and corrected for the effect of the fuel pellets. The model expands the rod displacement of Eq. (7) in terms of the unconstrained beam modes of vibration and solves the resulting Ordinary Differential Equation (ODE) system obtained by using the orthonormality relations of the modes. The rod motion and impact forces against the supports are then determined by numerically integrating the ODE system in a time domain simulation. 


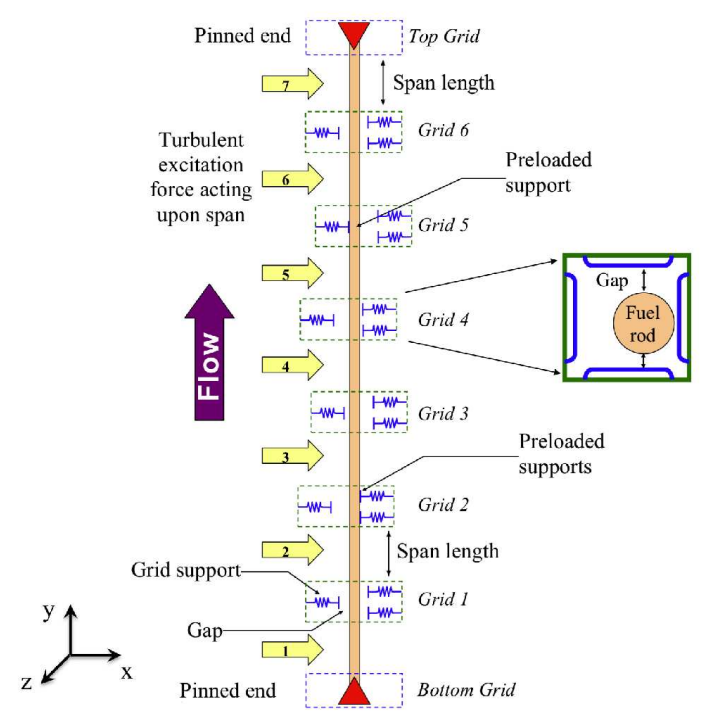

Figure 4: VITRAN model with full-length fuel rod, seven spans (yellow arrows), and six spacer grids (springs).

The fuel rod assembly grids are modeled as nonlinear constraining forces that are activated anytime the rod reaches the limit of available clearance inside a grid cell as shown in Figure 5. The support forces are decomposed into a) the normal or impact force and b) the tangential or frictional force. The normal force is calculated by modeling the support as a spring-damper where

$$
f_{s}^{\text {normal }}=\left\{\begin{array}{cl}
\operatorname{sign}\left(d_{n}\right) \cdot k_{s} \cdot\left(\text { gap }-\left|d_{n}\right|\right), & \text { if }\left|d_{n}\right| \geq \text { gap } \\
0, & \text { if }\left|d_{n}\right|<\text { gap }
\end{array}\right.
$$

where $d_{n}$ is the rod displacement in the normal direction to the support, gap is the distance between the support and the rod, and $k_{s}$ is the support stiffness. The support dynamic stiffness is experimentally measured and corrected for temperature effects.

The axial turbulent fluid forces acting upon the fuel assembly are modeled as external forces considered to be independent of the rod motion. This approach is justified since fluid-elastic instabilities have not been observed under the primary axial flow in the VIPER experimental test loop [24], and have not been observed during normal reactor operation conditions. The PSD of the axial turbulent flow can be estimated from an experimental PSD correlation developed for fuel bundles, and subsequently benchmarked by fuel rod vibration amplitudes measured in hydraulic loop tests. By approximat- 
Centered cell

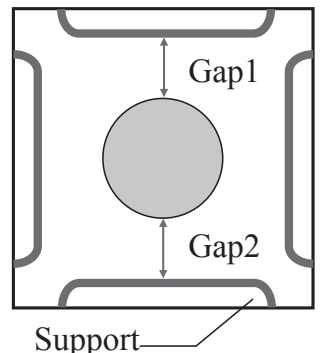

Support-
Zero gap cell

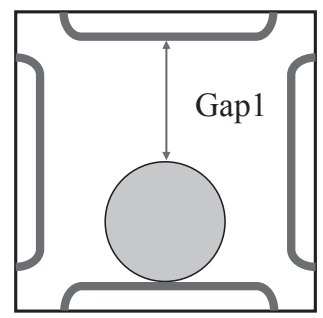

cell clearance $=$ gap $1+$ gap 2
Preloaded cell

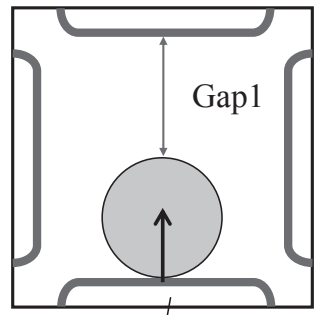

Preloaded

support

Figure 5: Three possible configurations of the fuel rod inside a grid cell.

ing the turbulence forces as a Gaussian "Band-Limited White Noise" and applying an inverse Fast Fourier Transformation (FFT) on the spectral components, the flow forces acting upon the rod during the transient simulation are calculated using the white noise and RMS amplitude.

VITRAN can be used to analyze different types of systems through an appropriate selection of the beam boundary conditions, the grid position, the support characteristics and the external forces distributions. An example of a fuel rod model for a six "mid-grid" assembly considered in this study is shown in Figure 4. In the example, the spacer grids have different conditions: Grid 1, 3, 4 and 6 have gaps between the rod and the supports, while the supports of Grid 2 and Grid 5 are touching the rod and thus a preload on the fuel rod exists.

The code integrates the ODE system obtained from Eq. (7) in time, and determines numerically time-averaged values for the normal work on each support of the grid. The time-averaged volumetric wear work-rates are calculated by using a modified Archad's law and the following relation [25]

$$
V_{\text {wear }}(t)=\int_{0}^{t} W_{n}(\hat{t}) \cdot K(\hat{t}) \cdot d \hat{t}
$$

where $K(\hat{t})$ is the instantaneous cladding wear coefficient and $W_{n}$ the normal work-rate. The wear coefficients of grid supports are considered to be timedependent quantities affected by geometry of the support features and wear severity. 


\section{Results}

This section begins with an ILES validation study using a $5 \times 5$ rod bundle with a Westinghouse spacer grid design. Following the validation study, results for a $3 \times 3$ rod bundle are presented and the sensitivity of the RMS force distribution downstream of the mixing vane are discussed. Finally, results from VITRAN studies using RMS force data from the $3 \times 3$ rod bundle ILES study are presented.

\section{1. $5 \times 5$ Rod Bundle Validation Study}

The $5 \times 5$ fuel rod bundle geometry was provided by Westinghouse, and corresponds to the experimental configuration used at Texas A\&M where PIV measurements were carried out. The flow domain is shown in Figure 6(a). Not shown here are the exterior walls of the flow housing used in the experimental facility. Additional details on the experimental configuration and results may be found in Conner, et al. [26].

Flow in the domain was forced by prescribing the inlet velocity, $\mathbf{v}=$ $(0.0,2.48,0.0)^{T} \mathrm{~m} / \mathrm{s}$, in conjunction with a zero pressure boundary condition at the outlet. With coolant properties set to correspond to those for water at $24^{\circ} \mathrm{C}$, this resulted in a Reynolds number, based on the hydraulic diameter of the rod bundle, of $R e_{D_{h}}=28,000$. The hydraulic diameter is defined as $D_{h}=4 A_{\text {flow }} / P_{\text {wet }}$, where $A_{\text {flow }}$ is the cross-sectional flow area and $P_{\text {wet }}$ is the wetted perimeter for the rod bundle. At the surfaces of the flow housing, rods, support and spacer grids, no-slip and no-penetration velocity conditions were prescribed.

For all the computations, a time-stepping algorithm was used wherein the time step was modified in order to maintain a maximum $C F L$ number, $C F L_{\max }=4$. The flow domain was meshed using Numeca's Hexpress/Hybrid mesh generator (a.k.a., Spider) using the meshing guidelines outlined in $\S 2$. The surface meshes for the spacer grid and fuel rods are shown in Figure 7(a) and (b). Two meshes were used, a coarse mesh with 14 million (14M) elements, and a finer one with 96 million (96M) elements. Both meshes were hex-dominant, but also required a mixture of tetrahedra, wedge and pyramid elements to close the complex geometry of the spacer grid and the region between the fuel rods and mixing vanes.

Following the computational workflow outlined in $\S 2$, a series of preliminary coarse-mesh calculations were performed. From these computations, it 


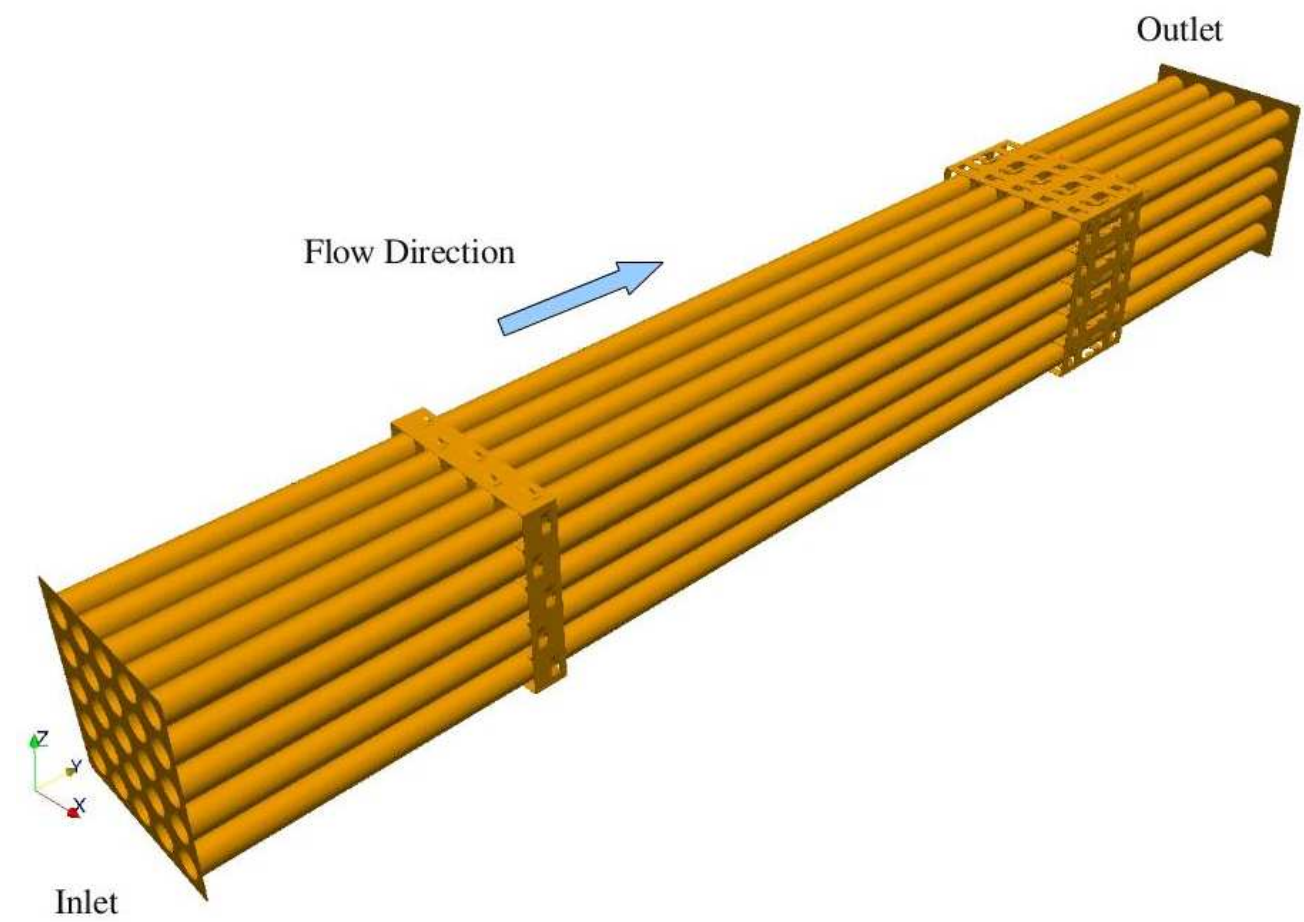

(a) Flow domain.

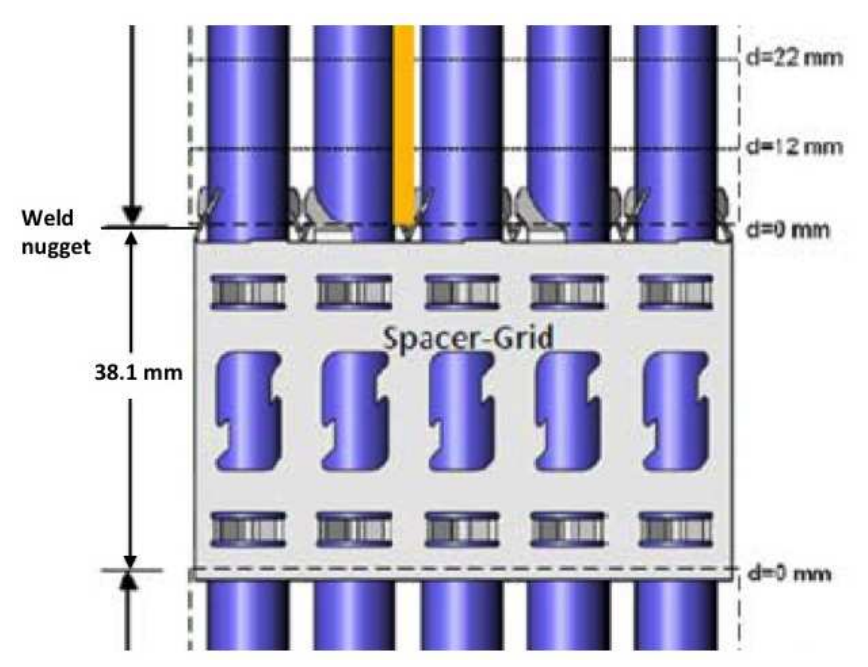

(b) Weld nugget location.

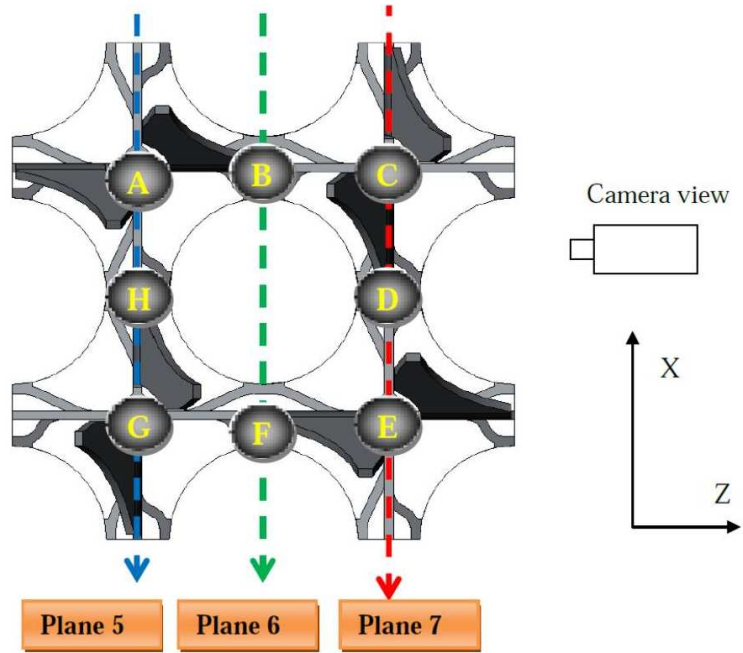

(c) Sample points.

Figure 6: (a) Flow domain showing rod bundle, spacer grid and mixing vanes, (b) locations for PIV measurements relative to the "weld nugget" used for extracting data along planes 5, 6 and 7, (c) sample points for PIV data. 


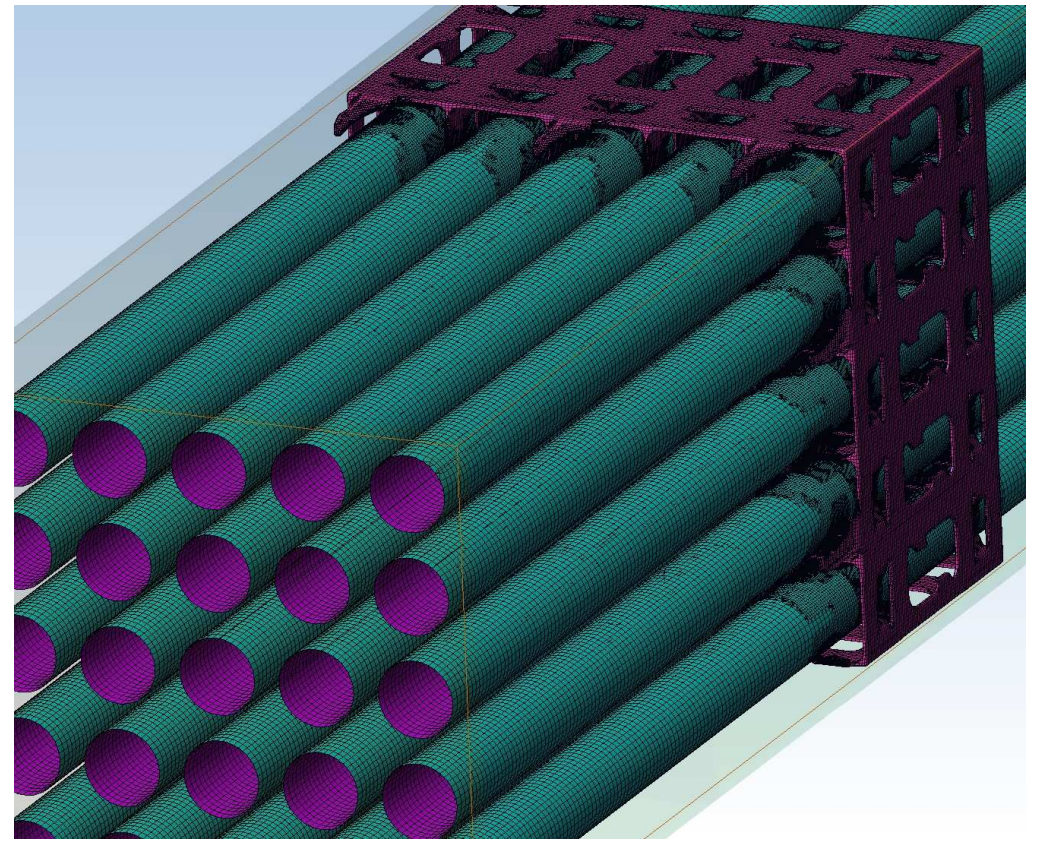

Figure 7: Surface mesh for the spacer-grid, and mixing vanes, and fuel rods.

was determined that the flow spun up and settled into a robust statisticallystationary turbulent state by 0.2 seconds. A total simulation time of 1 second was used, with flow statistics collected over a period of 0.8 seconds. Figure 8 shows a snapshot of the instantaneous helicity $(\mathbf{v} \cdot \omega)$ on the $96 \mathrm{M}$ mesh after the flow has become statistically stationary.

In order to compare to the experimental data presented in Yan, et al. [27], a series of line plots were extracted from the time-averaged velocity field for the $96 \mathrm{M}$ mesh. The locations for the line plots were selected to fall in the planes of the PIV measurements relative to the "weld nugget" as shown in Figure 6(b) and (c). The "weld nugget" is located at $38.1 \mathrm{~mm}$ from the bottom of the spacer grid [28]. The time-average for the computational results was over 0.8 seconds, while the experimental velocities were ensemble averaged with a window size of 0.8 seconds. Details on the experimental measurements are also presented in Yan, et al. [27].

Following Yan, et al. [27], mean velocities were compared at points A, C, D, E, G and H. For brevity, we have presented velocity comparisons at point $\mathrm{A}$ and $\mathrm{H}$ for the $96 \mathrm{M}$ mesh in Figure 9 . The stream-wise velocity in 


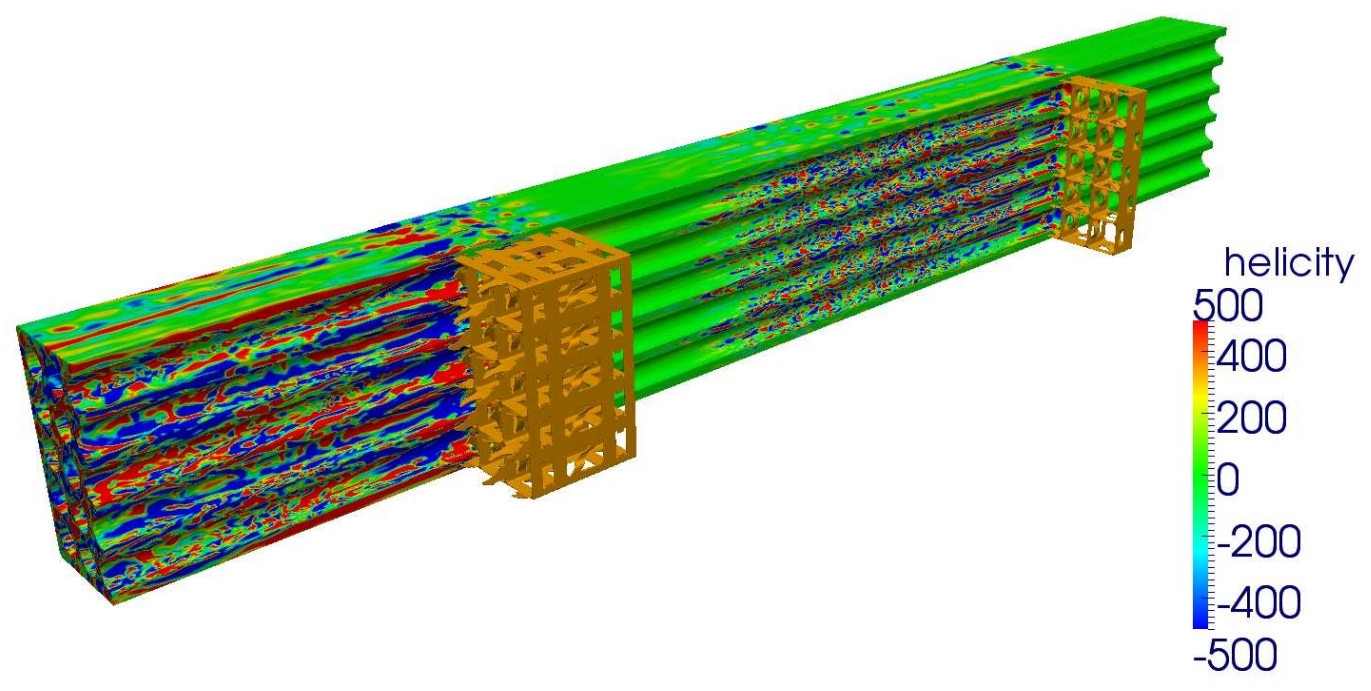

Figure 8: Snapshot of the instantaneous helicity field $\left(\mathrm{m} / \mathrm{s}^{2}\right)$ for the $96 \mathrm{M}$ element ILES.

the experiment corresponds to the y-velocity in the computation, while the lateral velocity corresponds to the x-velocity. In Yan, et al. [27], the systematic uncertainty in the velocities due to the PIV measurements, software data acquisition, etc., was estimated to be a maximum of $0.199 \mathrm{~m} / \mathrm{s}$. The statistical uncertainty, which is a function of the number of snapshots of the velocity, is estimated to be $0.414 \mathrm{~m} / \mathrm{s}$ in the lateral direction, and $0.372 \mathrm{~m} / \mathrm{s}$ in the axial direction with $v_{\text {inlet }}=2.48 \mathrm{~m} / \mathrm{s}$. All experimental data have been plotted with the uncertainty bounds provided by Dominguez-Ontiveros and Hassan (see also [26]). Our goal is that the ILES results fall entirely in the bounds of the experimental uncertainty. While the $96 \mathrm{M}$ results compare reasonably well to the experimental data, it was subsequently determined that the experimental uncertainties did not include the effect of positioning errors in the PIV traversal mechanism. Further studies to resolve this discrepancy have not been undertaken at this time.

Experimental time-averaged velocities in the plane from location $\mathrm{G}$ to $\mathrm{F}$ are shown with the computed time-averaged ILES velocity fields in Figure 10. Here, the peak velocities in the axial direction are slightly under-predicted, while the lateral velocities are slightly over-predicted in the Hydra-TH com- 
Position A

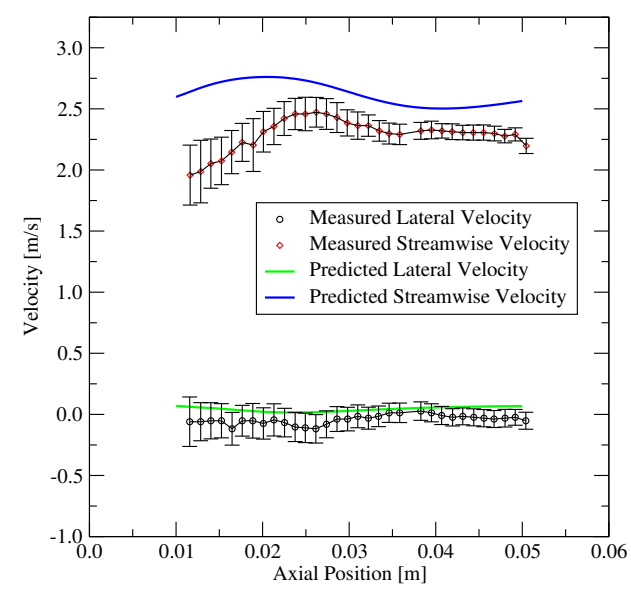

(a) 14M Velocities at A.

Position A

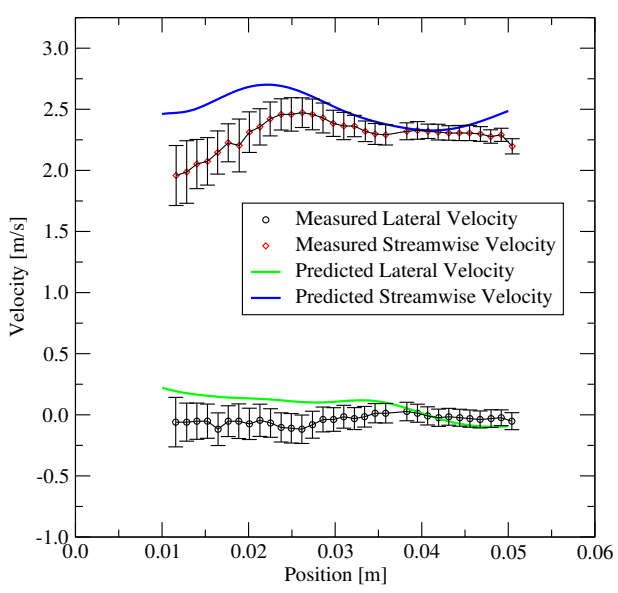

(c) $96 \mathrm{M}$ Velocities at A.
Position $\mathrm{H}$

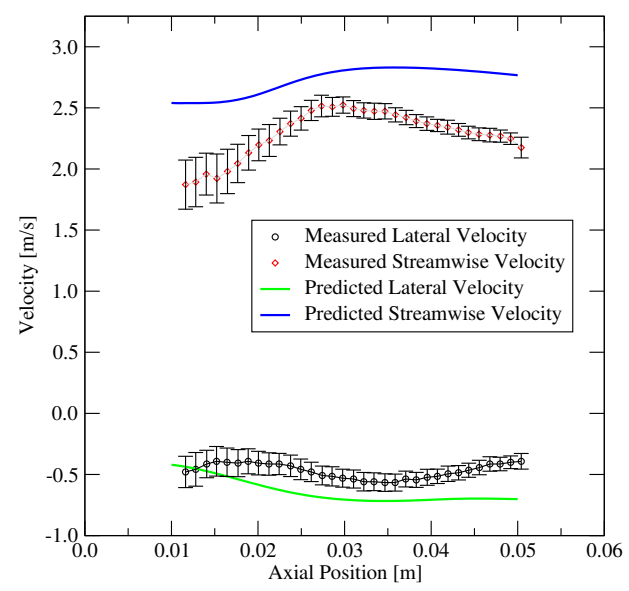

(b) 14M Velocities at $\mathrm{H}$.

Position $\mathrm{H}$

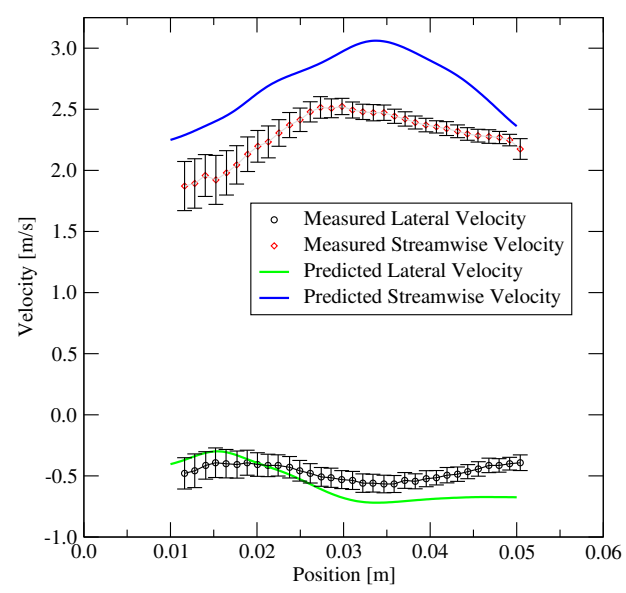

(d) $96 \mathrm{M}$ Velocities at $\mathrm{H}$.

Figure 9: Measured and predicted mean axial and lateral velocity profiles at positions A and $\mathrm{H}$ for the $14 \mathrm{M}$ and $96 \mathrm{M}$ meshes. 
putations. We note that the experimental data presented here was computed using a $50 \times 40$ grid for time-averaging resulting in a somewhat discrete appearance in Figure 10 (a) and (c). However, because only the measured velocity vectors are used for time-averaging, the minimum/maximum velocities are based on actual velocity measurements rather than interpolation.

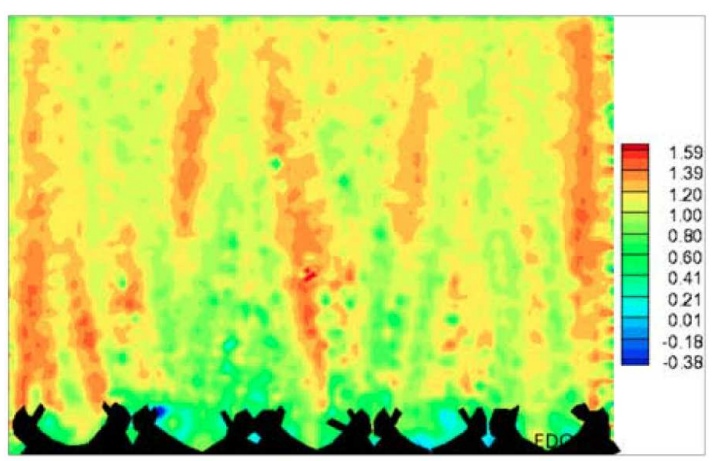

(a) Measured Axial Velocity

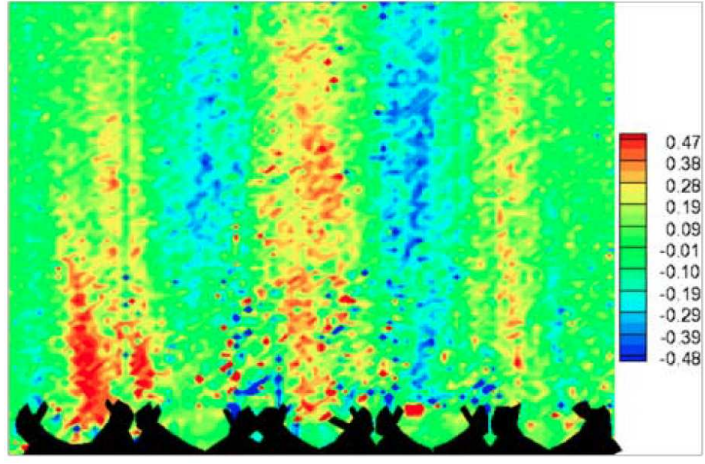

(c) Measured Lateral Velocity

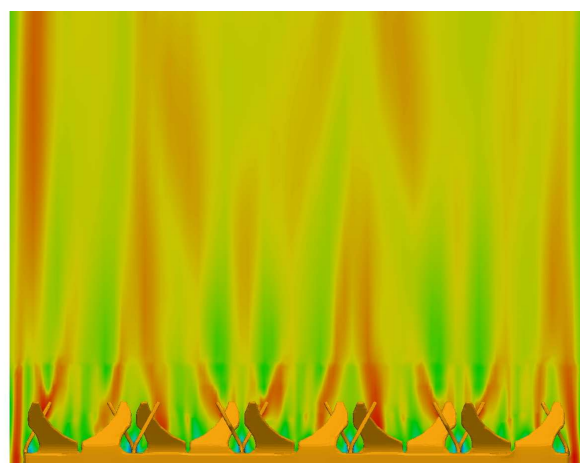

(b) Predicted Axial Velocity

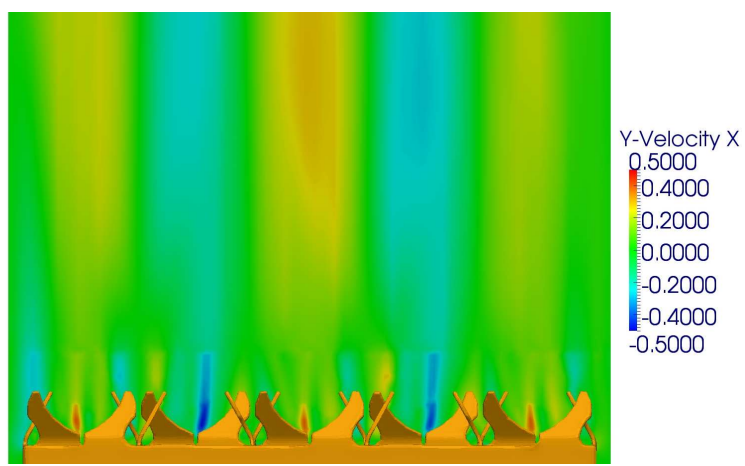

(d) Predicted Lateral Velocity

Figure 10: Measured and predicted axial (y-direction) and lateral (x-direction)timeaveraged velocities on plane $\mathrm{G}-\mathrm{E}$. The velocity magnitude has been scaled relative to the $2.48 \mathrm{~m} / \mathrm{s}$ inlet velocity.

\section{2. $3 \times 3$ Rod Bundle Simulations}

A Westinghouse $3 \times 3$ spacer grid design is considered on a single span of a fuel assembly. A series of ILES calculations using meshes with 2, 7, 14, and 27 million cells (2M, 7M, 14M and 27M respectively) have been carried out. Figure 11 shows a representative surface mesh of the central fuel rod 
and spacer grid and mixing vanes. For these grids, the wall resolution was such that $0.72 \leq y_{\min }^{+} \leq 1.55$, and $43.18 \leq y_{\max }^{+} \leq 73.41$, with the larger wall-distance occurring in regions away from the spacer grid.

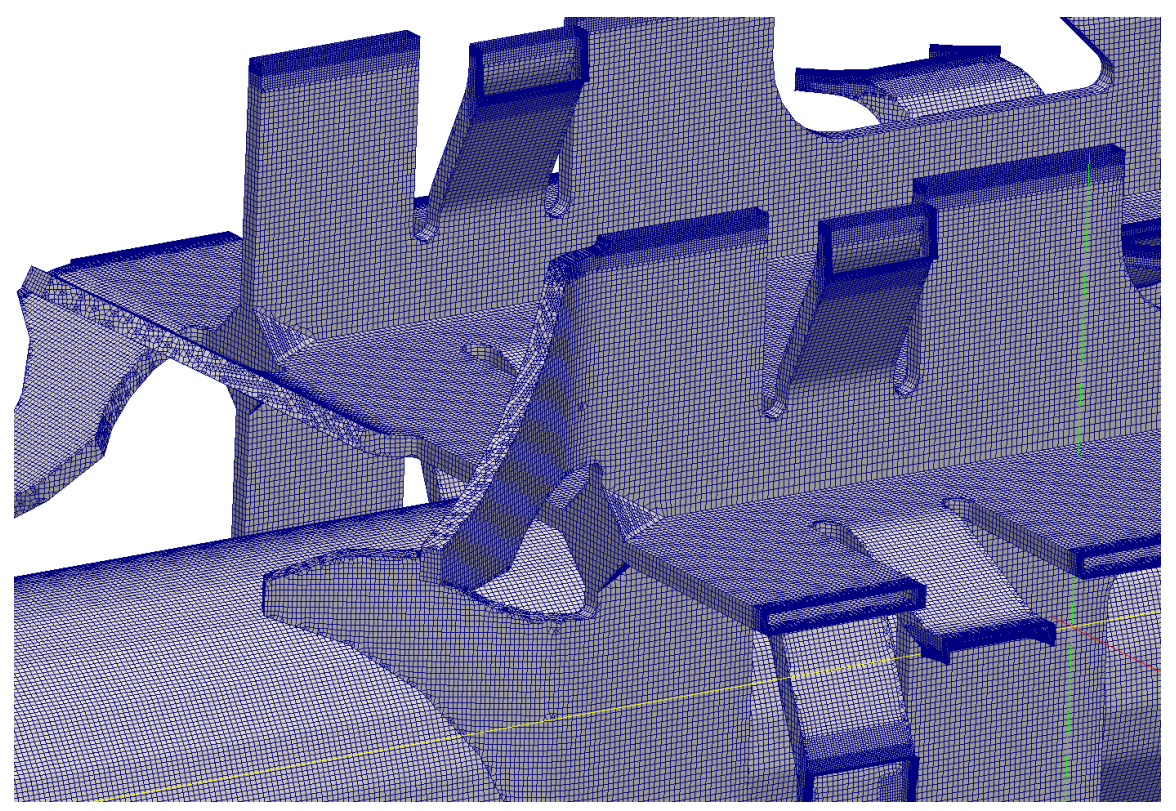

Figure 11: Surface mesh for the center rod, spacer grid and mixing vanes in the $3 \times 3$ rod bundle.

For the $3 \times 3$ calculations, the working fluid is water at a temperature of $394.2 \mathrm{~K}$, with a density of $942.0 \mathrm{~kg} / \mathrm{m}^{3}$, and a dynamic viscosity of $2.32 \times 10^{-4} \mathrm{~kg} / \mathrm{m} / \mathrm{s}$. The inlet velocity is prescribed as $\mathbf{v}=(0,5,0)^{T} \mathrm{~m} / \mathrm{s}$. This corresponds to a Reynolds number, based on the rod diameter, of $R e_{D}=1.93 \times 10^{5}$, while the Reynolds number based on the hydraulic diameter is $R e_{D_{h}}=4.01 \times 10^{5}$. No-slip, no-penetration boundary conditions are prescribed at the rod and spacer surfaces. At the outflow, the hydrostatic pressure is specified to be $p_{h}=0.0 \mathrm{~Pa}$. No-penetration conditions with in-plane slip were applied at the subchannel boundaries.

The work by Elmahdi, et al. [29] serves as a point of reference for these calculations. In this work, STAR-CCM+ was used with a hex-dominant mesh consisting of $48 \mathrm{M}$ cells. Here wall functions were used with an unspecified LES closure. The mesh resolution at the wall was such that $40 \leq y^{+} \leq 60$. For the CCM+ computations, $C F L_{\max }=1.8$ was used. Otherwise, all other flow parameters were identical to those used here. 
In the ILES computations, and the work by Elmahdi, the section of the central fuel-rod downstream of the mixing vane was subdivided into 12 1inch long segments where the time-dependent fluid forces were computed. In the ensuing discussion, these 1-inch segments are numbered 1 through 12 increasing in the flow direction. The Hydra-TH ILES computations were highly instrumented recording time-series data of the wall shear, pressure and total forces on the 1-inch segments and spacer grid, as well as wall shear, $y^{+}$, and traction on all no-slip/no-penetration surfaces. In addition, time-series data of velocities and global kinetic energy were used to monitor the health of the computations. Derived statistics included means of all the primitive variables, wall shear, and high-order statistics including RMS pressure, velocity, turbulent kinetic energy and Reynolds stresses.

As before, sensitivity studies have been performed to assess when a statistically stationary flow exists, and the duration of time required for accurate statistics to be determined. Statistical stationarity is established at approximately 0.1 seconds, and time-averaging was carried out for 0.9 seconds. Figure 12 shows isosurfaces of the instantaneous helicity field downstream of the spacer grid. Here, the role of the spacer grid and mixing vane as a turbulence generator is clear. The propagation of coherent flow structures far downstream shows that the neutrally dissipative algorithm in Hydra-TH does an excellent job of accurately representing the complex vortical structures.

Figure 13(a) shows a line where the instantaneous and time-averaged (mean) pressure is sampled. The pressure along the axial flow direction for the four meshes along the line are compared in Figure 13(b). The vertical dashed lines in the pressure profile figures identify the bounds of the upstream spacer grid and the downstreawm mixing vanes. It is reassuring that the pressure lines are qualitatively very similar for all mesh resolutions. Since the pressure at the outflow is fixed at $p=0 P a$, the value that the inlet pressure achieves yields the overall pressure drop for the flow domain.

The time-averaged mean pressure along the rod is plotted in Figure 13(c). A large drop in the mean pressure through the spacer indicates that most of the pressure loss is due to the spacer as expected. In spite of the turbulent mixing induced by the spacer the characteristic peaks and troughs in the profile of the mean pressure are reproducible throughout the spacer using the $2 \mathrm{M}, 7 \mathrm{M}, 14 \mathrm{M}$ and $27 \mathrm{M}$ meshes. Downstream of the mixing vanes a slight wave in the mean pressure is apparent from the ILES on the coarsest 2Mmesh suggesting an under-resolved calculation. However, the mean pressure using the $7 \mathrm{M}, 14 \mathrm{M}$ and $27 \mathrm{M}$ meshes appears to be what one would intuitively 


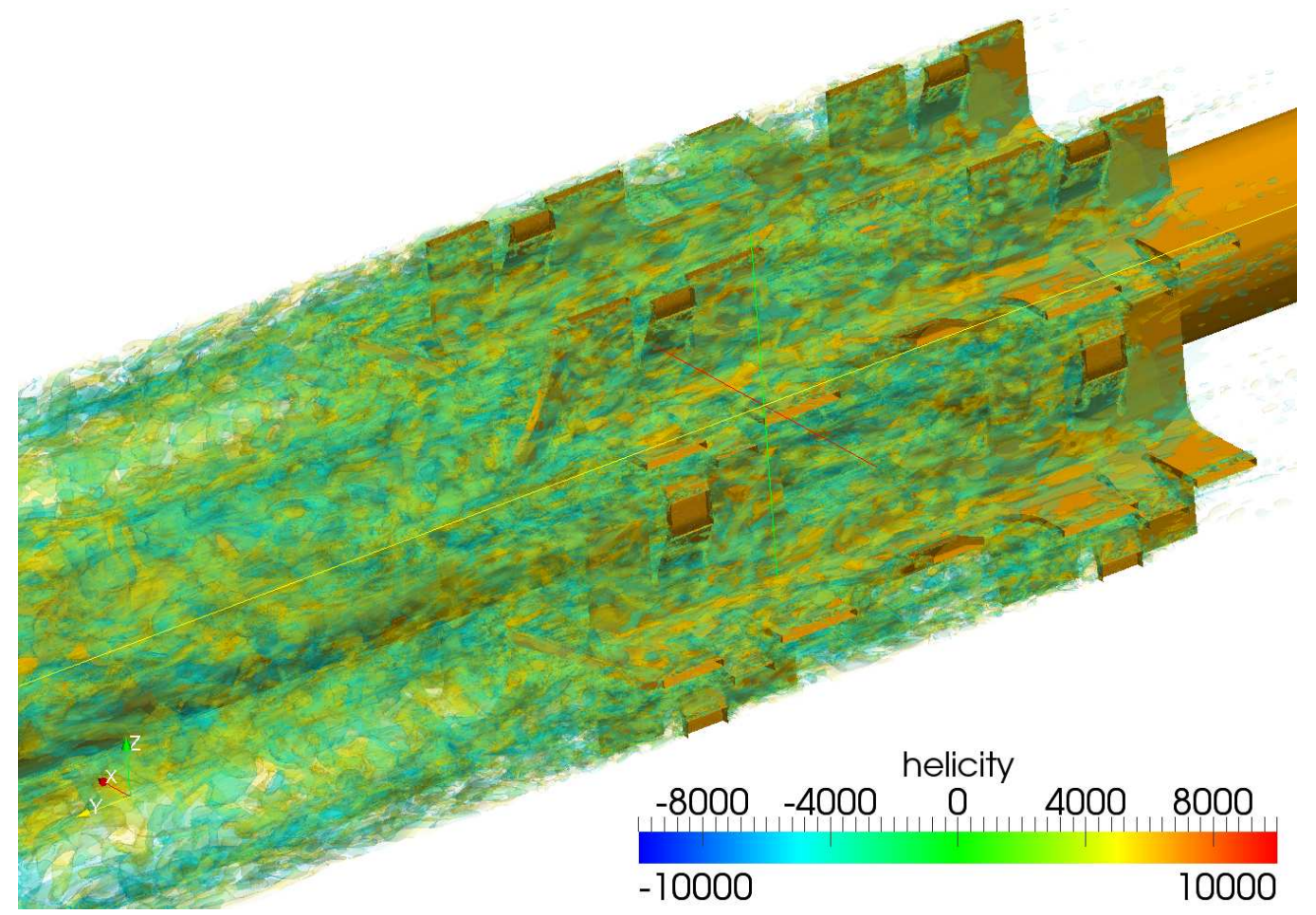

Figure 12: Snapshot of instantaneous helicity downstream of the $3 \times 3$ spacer grid. Transparent helicity isosurfaces are shown at $\pm 4000 \mathrm{~m} / \mathrm{s}^{2}$

expect for a turbulent channel flow, i.e., the mean pressure linearly decreases along the axial flow direction.

The RMS pressure along the rod is plotted in Figure 14(a) for the 2M, $7 \mathrm{M}, 14 \mathrm{M}$ and $27 \mathrm{M}$ meshes. The fluctuating pressure force is likely the most important quantity to accurately compute a reasonable representation of the RMS forces acting on the fuel rods and the concomitant fretting wear. Figure 14(a) shows that the RMS pressure peaks at the downstream end of the spacer for all meshes. This is expected, since this is where the level of turbulent kinetic energy is the largest. While the downstream locations of the peaks are somewhat aligned for the two meshes, their amplitudes and downstream evolution are quite different. Once again, we find the $2 \mathrm{M}$ mesh is too coarse to adequately capture the RMS pressure.

Additional insight into the fluctuating velocity field is given in Figure 14(b) where the spatial evolution of the turbulent kinetic energy (TKE) 


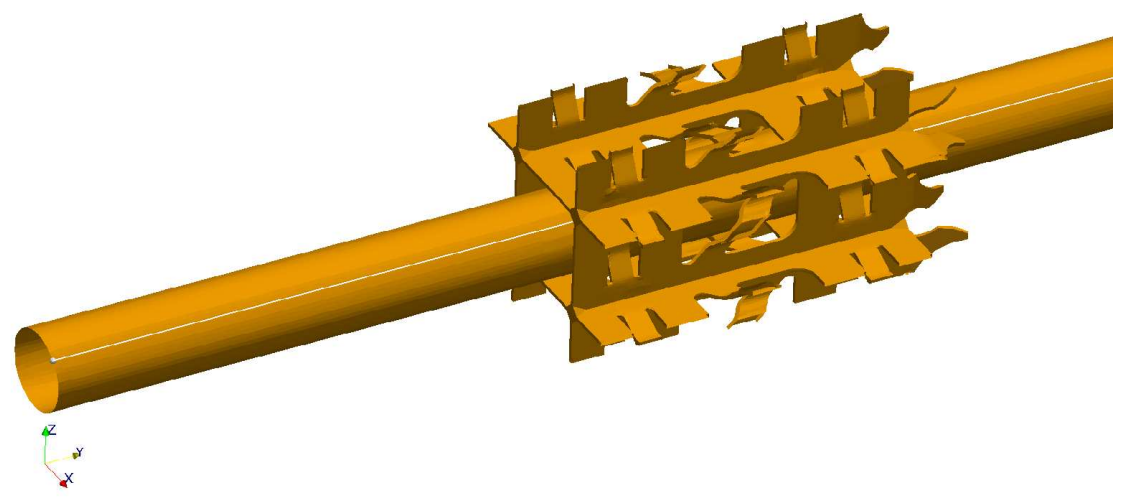

(a) Line for pressure profile comparison

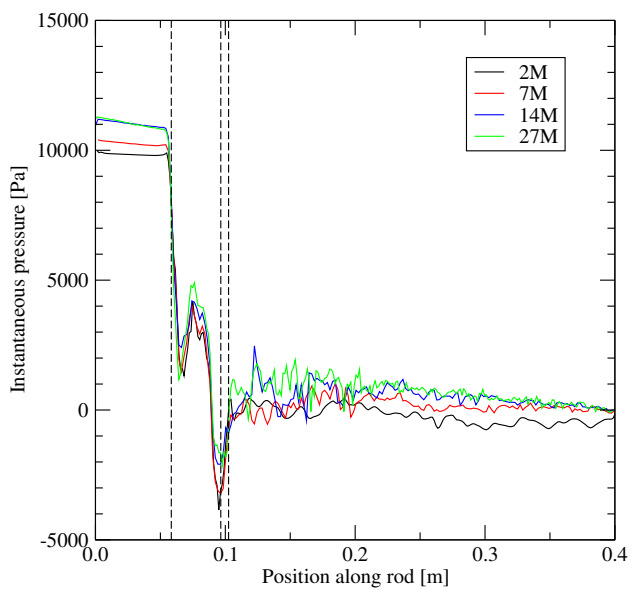

(b) Instantaneous pressure

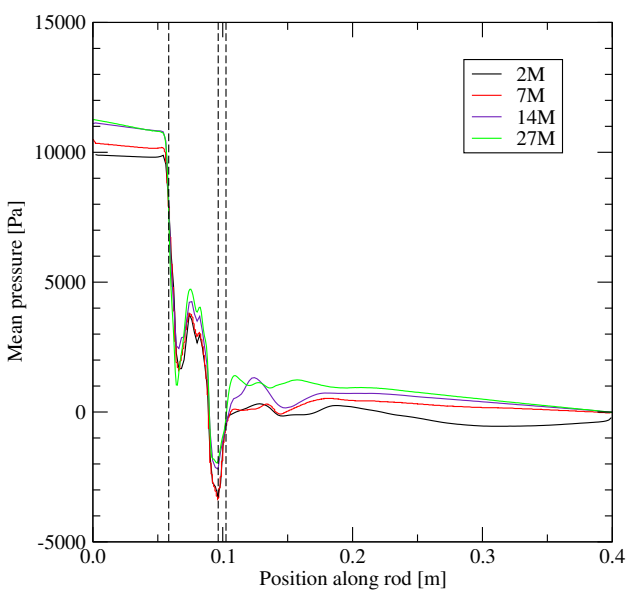

(c) Time-averaged pressure

Figure 13: Axial pressure profiles for the $3 \times 3$ rod bundle: (a) white line near the rod surface where pressure is sampled, (b) instantaneous pressure along the line, (c) mean pressure along the line. 
is plotted. Similar to the pressure fluctuations in Figure 14(a), the TKE, $\frac{1}{2}\left\langle\mathbf{v}^{\prime} \cdot \mathbf{v}^{\prime}\right\rangle$, where $(\cdot)^{\prime}$ denotes the fluctuation about the mean, peaks in the vicinity of the mixing vanes and stays at a relatively high value until approximately $0.2 \mathrm{~m}$ downstream. This reinforces the earlier observation that the highest level of TKE occurs close to the downstream edge of mixing vanes. The under-resolved character of the 2M ILES is underscored by the behavior of the TKE downstream of the spacer grid. Figure 14(c) shows the downstream evolution of the different components of the Reynolds stress tensor for the intermediate resolution $14 \mathrm{M}$ mesh. The figure shows that the flow downstream of the mixing vanes remains highly anisotropic until the end of the computational domain. A significant amount of the kinetic energy is in the stream-wise $(y)$ component, i.e., the stream-wise fluctuations are large when compared to both cross-stream components.

Having performed simulations at four different mesh resolutions, we consider Richardson's deferred approach to the limit (of infinite resolution) [30]. Sometimes also simply called Richardson's extrapolation (RE), this wellestablished procedure is capable of significantly improving the accuracy of numerical solutions of differential systems, as in the present case, with only post-processing. Alternatively, we could have continued to increase the mesh resolution seeking a "mesh-independent" solution via brute force, but the mesh and computing requirements would be significant. Instead, we chose to use RE, a knowledge of the behavior of our numerics and the statistics for the quantities of interest, e.g., RMS forces in with meshes that exhibit convergent behavior. This approach was taken specifically with industrial application in mind, where unlimited computing resources are not available, in order to assess the overall methodology.

Proceeding with RE, if the quantity to be estimated is $f(y)$, this procedure uses estimates of $f(y)$ at two or more resolutions, represented as $F(y ; h)$ where $h$ is a characteristic mesh size under the assumption that the known $(F(y ; h))$ and unknown $(f(y))$ estimates are related as

$$
F(y ; h)=f(y)+k_{2}(y) h^{2}+k_{4}(y) h^{4}+\ldots+k_{2 n} h^{2 n}+\ldots
$$

Here $n \geq 1$ is the number of levels of refinement at which estimates of the quantity of interest are available, from having performed the full simulations at those resolutions.

The qualitatively different nature of the $2 \mathrm{M}$ element mesh solution in Figure 14(a) suggests that the resolution is too coarse, and the solution on 


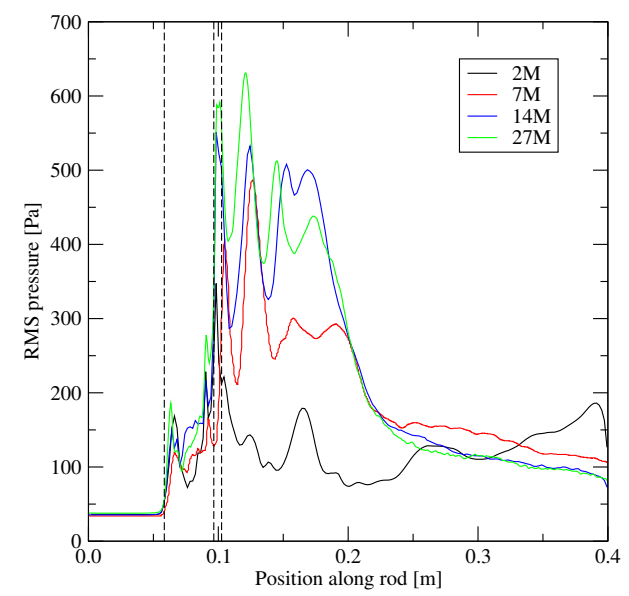

(a) RMS pressure

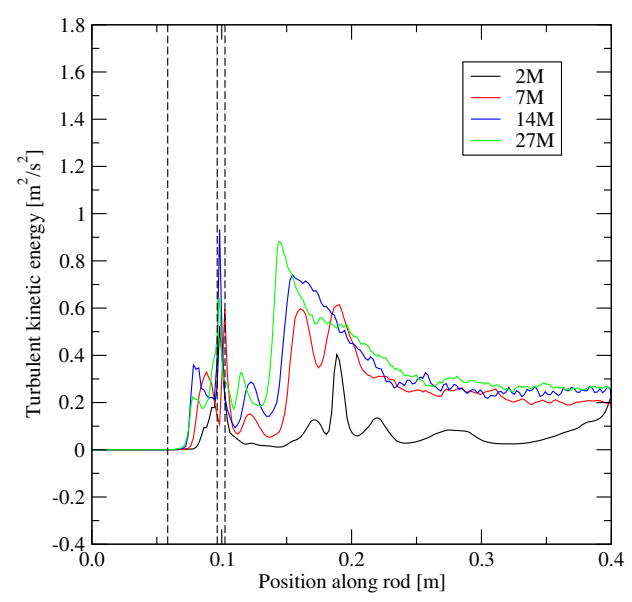

(b) Turbulent kinetic energy

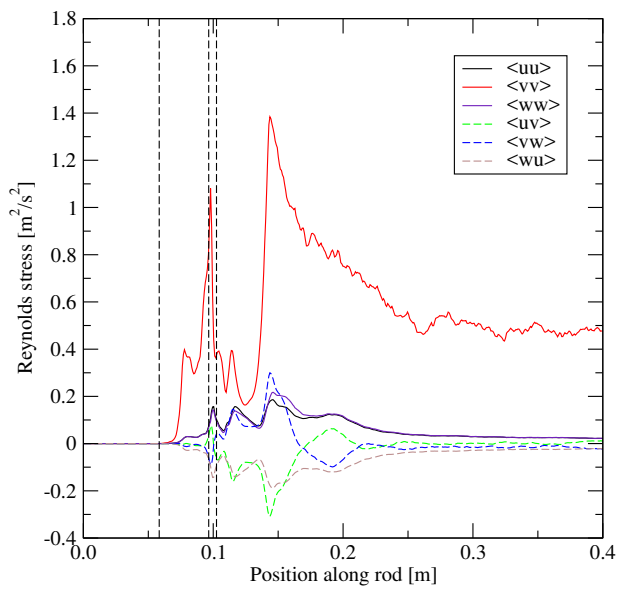

(c) Reynolds stress (27M mesh)

Figure 14: Profiles of (a) RMS pressure, (b) turbulent kinetic energy, and (c) Reynolds stress. 
the $2 \mathrm{M}$ element mesh has not entered the asymptotic regime, represented by Eq. 10, and required for the application of Richardson's extrapolation. For this reason, the $2 \mathrm{M}$ mesh results are not included in the Richardson's extrapolation exercise.

Figure 15 shows the axial profile of total force acting on the central rod integrated in 1-inch segments in the $x$-direction (left panel) and in the $z$ direction (right panel). In addition to showing the forces at the four resolutions considered, this plot also shows the value of the forces obtained by the Richardson's extrapolation procedure and that obtained in the LES computations of Elmahdi [29] that used STAR-CCM+ with a $48 \mathrm{M}$ element mesh.

There are a number of features worth noting in Figure 15. First, as in Figure 14(a), it is seen that the solution using the $2 \mathrm{M}$ element mesh has not reached the asymptotic range where the solution-derived quantities may be represented as in (10). Next, the Richardson extrapolated values are seen to reiterate and confirm the convergence properties of the Hydra-TH solutions at the refined resolutions of 7M, 14M, and 27M elements. For this, it may help to note that the (largely) monotonic behavior of the Hydra-TH solutions seen as a function of resolution switches between the second and fourth segments. Finally, in a least-squares sense, the Elmahdi force values are closest to the 27M element Hydra-TH forces. Regardless of this, in order to make the comparison rigorous, we attempted an estimation of error-bars on the Hydra-TH computations, while noting that we do not have a basis to estimate error-bars for the Elmahdi force values.

A comprehensive assessment of the Hydra-TH computations performed for this problem suggests that uncertainty due to boundary and and initial conditions are small. We also spin the flow up to a robust statistically stationary state before collecting force statistics. Further, and in part aided by the Richardson's extrapolation procedure above, it is reasonable to think that uncertainty due to resolution is small as well. The remaining sources of uncertainty, then, lie in the turbulence model and in the estimation of the RMS of the forces themselves. It seems likely that the uncertainty related to the turbulence model structure and turbulence parameter values is more important. That is unfortunately, however, beyond the scope of the present article. Although the latter source of uncertainty, related to the estimation of RMS of the forces, can be driven to zero by considering yet longer simulations, we computed the error-bars for the data from the one second runs that went into the estimates of the forces in Fig. 15. 
The primary reason to expect uncertainty in the estimate of RMS of the force on the central rod in the finite length ILES computations is related to the intermittent nature of turbulence - an aspect of turbulence that can lead to an increase in the low-frequency variability of the system. For example, it is conceivable that with increasing resolution, turbulence intermittency increases, leading to increased spectral power at low frequencies.

The auto-correlation of the force time series (as a function of time lag) or equivalently the PSD is a useful tool in gauging uncertainty in the estimate of the RMS of the forces that are key input to further rod vibration analysis. For this reason, we first computed the auto-correlation of each of the force time series. When there is a separation of scales, it is rather straight-forward to estimate a decorrelation scale using the auto-correlation function. For example, in such a case, estimates of the decorrelation time are provided by a) the first zero crossing of the auto-correlation function, or b) an integral of the auto-correlation function such as

$$
\tau_{D}=\int_{0}^{\infty} \mathcal{A}(\tau ; F(y ; h)) d \tau
$$

where $\mathcal{A}(\tau ; F(y ; h))$ is the auto-correlation function of $\mathrm{F}(\mathrm{y} ; \mathrm{h})$ at lag $\tau$. Both these estimates for the $z$ - component of the force on each segment is shown in Fig. 16. (The corresponding behavior for the $x$-component is similar.) This figure shows that the two estimates of decorrelation time differ by about two orders of magnitude, and we find this somewhat surprising.

It is generally thought (e.g., see [19]) that the estimate of decorrelation time provided by the integral in Eq. 11 is more robust and more representative. However, the fact that this estimate differs from the zero-crossing estimate by about two orders of magnitude led us to further analyze the time series. Indeed, we find that the integral estimate is poorly behaved (i.e., highly variable) with respect to changes to the upper-limit in Eq. 11. For this reason, we suspect that the "zero-crossing" estimate is a better estimate under the present circumstances. We also note that the "zero-crossing" estimate correlates better with the maximum of the above integral (as a function of the upper limit of the integral).

Given the wide range of estimates of decorrelation time above, we also conducted spectral analysis of the force time series to gain insight into timescales of the system. For example, averaged periodogram estimates of power spectral density for the $z$-component of the force on the second segment is shown at the four resolutions considered in Fig. 17. In these figures, the 
uncertainty of estimates at the lower of the frequencies is reduced by segmenting the full time series and averaging the windowed spectral estimates. For this reason, the lowest frequency at which power is estimated is limited to about $25 \mathrm{~Hz}$. Features worth noting in this plot include the following: a) The spectral characteristics of the $2 \mathrm{M}$ mesh resolution forces are qualitatively different from that at higher resolutions. b) The broadband nature of turbulence is evident at the $7 \mathrm{M}, 14 \mathrm{M}$, and $27 \mathrm{M}$ mesh resolutions. Furthermore, it is seen that the temporal intermittency characteristics of the RMS of the integrated force due to turbulence at these resolutions is quite similar and stable. c) The fastest processes have a frequency of about $6000 \mathrm{~Hz}$. However, the energy contained at these frequencies is minute. d) And finally, and as previously discussed temporal intermittency of turbulent events leads to highest levels of energy at the lowest frequencies. Indeed, a more-or-less flat spectrum is seen over a significant range of low frequencies.

Next, we note that the frequency distribution of energy as in Fig. 17 provides a basis for gauging the importance of different timescales in the system. For example, the appearance of a floor in the spectra around $6000 \mathrm{~Hz}$ suggests that the corresponding time-period is the shortest characteristic time of the system. Further, on neglecting the estimate at the lowest frequency $(\approx 25 \mathrm{~Hz}$ ), the flatness of the distribution up to about $200 \mathrm{~Hz}$ suggests the importance of the corresponding period as another characteristic time scale of the system. Given the generic flatness of the frequency distribution of energy at low frequencies, in order to be able to objectively and easily identify such a characteristic time scale, we chose the lowest frequency at which $99 \%$ of the energy (variance of the force considered) was explained, and then repeated the same procedure for each of the force time series. (The analysis was repeated for a range of thresholds down to 90\%.) Indeed, the values of such a characteristic timescale were much closely related to the previous "zero-crossing" estimate of decorrelation time. We therefore think that in the present setting where we are interested in the low-frequency variability of the system as occurs due to intermittent turbulent activity, the "zero-crossing" estimate of decorrelation time is the right one to use.

Using the "zero-crossing" estimate of decorrelation time, the standard error in the estimate of standard deviation was approximated using the formula

$$
S E(\sigma)=\frac{\sigma}{\sqrt{2(n-1)}}
$$

Here $\sigma$ is the sample estimate of standard-deviation and $n$ is the number of 
independent samples, estimated here as $0.9 / \tau_{D}$. The resulting error bars are shown in Fig. 15. These error-bars, however, do not seem to help much in being able to suggest that the Elmahdi force values are in better agreement with a particular resolution of the Hydra-TH force values. But as we show in the next section, such a comparison can be made on further aggregating the forces on the twelve segments into three groups.

\subsection{Nonlinear Dynamics and Wear Work Rate}

The transient fuel rod forces resulting from the Hydra-TH ILES and the STAR-CCM+ LES are used as input forces for VITRAN to predict the fuel rod vibrational response, i.e., compute displacements, accelerations, and fuel rod wear. An issue that arises in this context is that of correlation between forces on adjacent rod segments. We note that Pearson's correlation coefficients between two adjacent segments as calculated by Elmadi, et al.[29] for the CCM+ segment forces show relatively weak correlations, with only adjacent rod segments displaying significant correlations. These correlation coefficients varied in magnitude from 0.11 to 0.44 . From the point of view of the setup used for computation of nonlinear dynamics of the fuel rod, it seems reasonable to combine two or more adjacent segments together: In particular, a sensitivity study was performed to assess dependence of the nonlinear fuel rod response to the number of segments (of a single fuel rod span) on which force was specified. Results from this study suggest that combining RMS forces on multiple rod segments has little impact on the rod vibration calculations and the wear work-rate. This result may be understood in light of the finding that the first few, and most dominant, vibrational modes of the full-span fuel rod have no high amplitude node points within the fuel rod span itself [24]. We also note that on so lumping forces, correlation between forces on the lumped segments was further reduced. For these reasons and to be able to make comparisons to previous computations, we follow Elmahdi, et al. [29] in combining the segment forces prior to the nonlinear dynamics analysis.

For completeness, details of how forces on the rod segments were combined is noted here: Three lumped forces are derived from the RMS forces computed on one-inch segments downstream of the spacer grid for use with Eq. (13). As previously mentioned, based on the dominant rod vibration modes the three lumped forces per fuel assembly span are sufficient. Each lumped force is the summation of several adjacent rod-segment forces. The lumped forces are located at 2 inches (segments 2-3), 4.5 inches (segments 


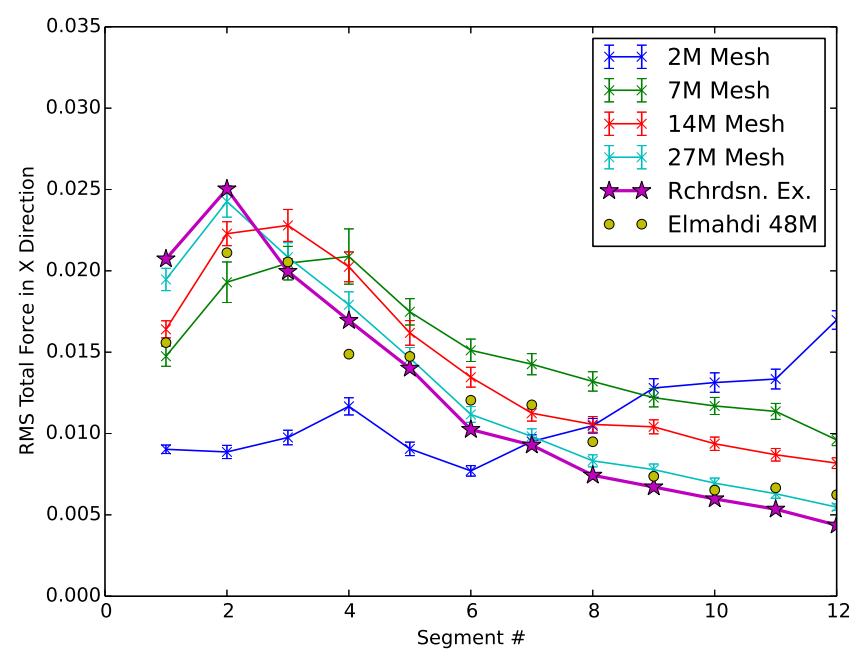

(a) RMS X-Force

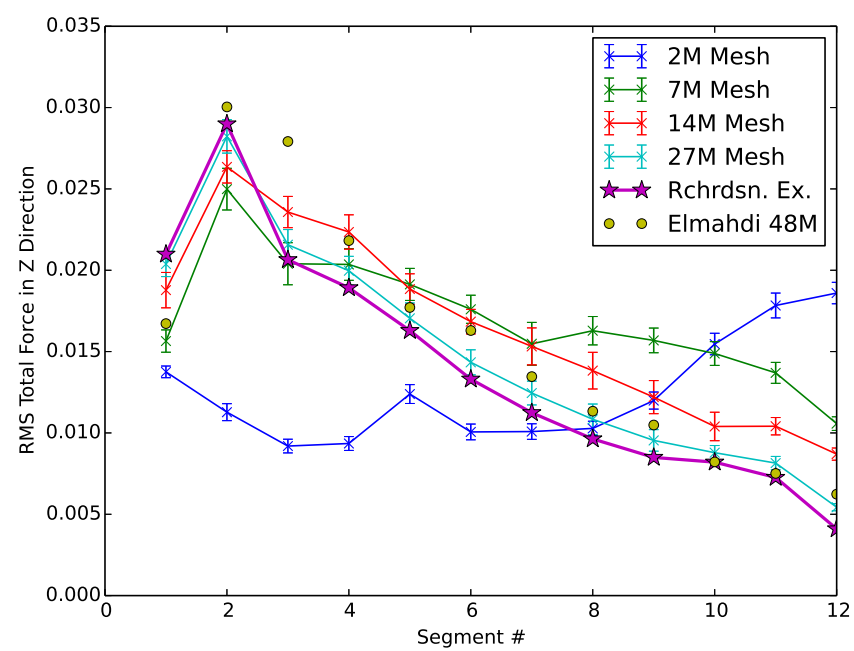

(b) RMS Z-Force

Figure 15: Axial profile of total force acting on the central rod integrated in 1-inch segments:(a) in the $x$-direction, (b) in the $z$-direction. The LES calculations of Elmahdi [29] use Star-CCM+ with a 48M hex-dominant mesh. 


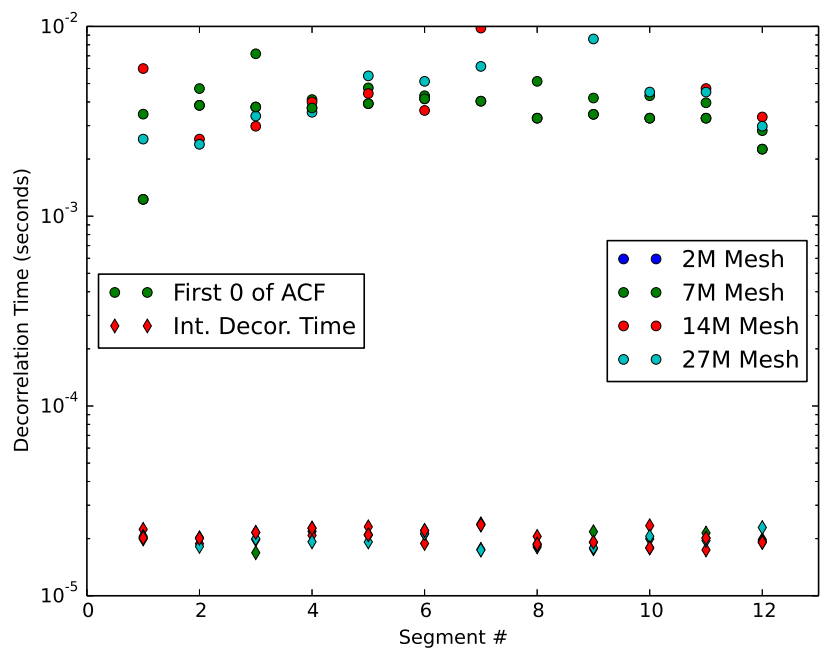

Figure 16: Two measures of decorrelation time scale for the force history in the LES simulations at four different resolutions. The decorrelation time scale related to the first zero-crossing of the auto-correlation function is shown using circles, whereas the integral measure is shown using diamonds. Estimates shown are for the $z$-component of the force. Those for the $x$-component are similar.

$4-6$ ), and 9 inches (segments $7-12$ ) downstream of the mixing vane in the VITRAN models.

Spectral analysis of GTRF test data [24] and earlier CFD simulation data [29] have shown that contributions from frequency components higher than $100 \mathrm{~Hz}$ are small. Indeed, the spectral analysis of forces on one of the segments in the Hydra-TH computations shown in Fig. 17 shows that power begins to drop at about $200 \mathrm{~Hz}$. Considering that the forces on individual segments are further aggregated (as discussed previously) before being used to drive the nonlinear structural dynamics computations, a spectral cut-off of $100 \mathrm{~Hz}$ for the VITRAN forcing seems consistent and reasonable. Furthermore, this allows us to compare results of the present Hydra-TH based computations to those obtained previously using STAR-CCM+. For these reasons and given the flatness of spectral distribution at low frequencies, a "Band-Limited Gaussian White Noise" form is chosen for the forcing in Eq. 7. If $F_{R M S}$ is the RMS of the lumped force resulting from the turbulent flow 


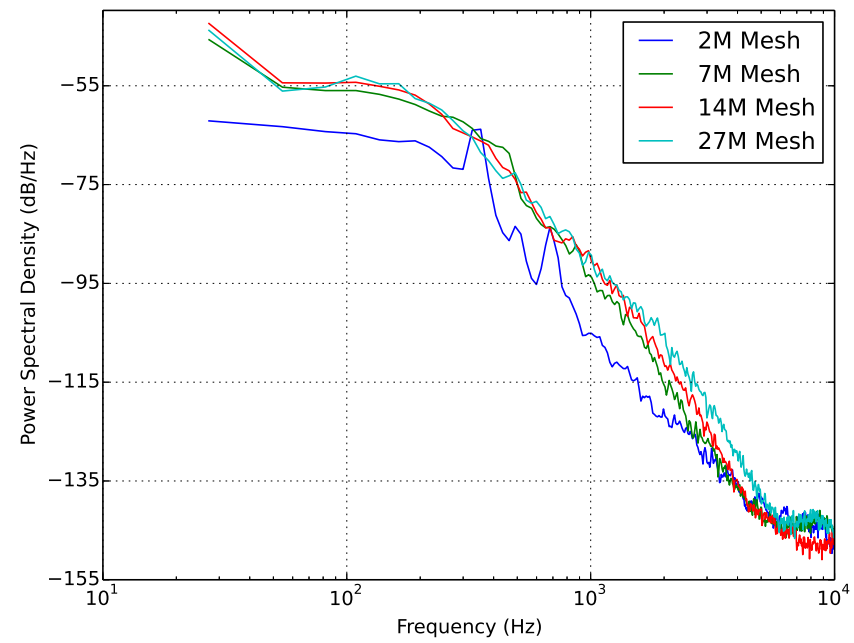

Figure 17: PSD estimates at the second segment at the four resolutions considered. Estimates shown are for the $z$-component of the force. Those for the $x$-component are similar.

computation, the magnitude of the Power Spectral Density is computed as

$$
P S D=\frac{F_{R M S}^{2}}{2\left(f_{\text {upper }}-f_{\text {lower }}\right)}
$$

where $f_{\text {lower }}(5 \mathrm{~Hz})$, and $f_{\text {lower }}(95 \mathrm{~Hz})$ are the cutoff frequencies. A specification of the PSD and the cutoff frequencies is then used to obtain a realization of the "Band-Limited Gaussian White Noise" force time series.

Using the RMS forces from the 7M, 14M, and 27M ILES calculation, Richardson extrapolation is performed. The lumped RMS forces forces are shown in Figure 18 for the 3 Hydra-TH calculations, the Richardson extrapolation, and STAR-CCM+. Similar to the results presented in Figure 15, under mesh refinement, the Hydra-TH results trend towards the RMS forces computed using Richardson extrapolation. Again, the lumped 48M CCM+ RMS forces are closest to those computed on a 14M mesh with Hydra-TH.

Figures 19 and 20 show the fuel rod acceleration and displacement computed with VITRAN for a 7-span fuel-rod bundle using the RMS forces from Hydra-TH, Richardson extrapolation, and CCM+ as input. The fuel rod spans are numbered from 1 to 7 in the flow direction as shown in Figure 4. Both the acceleration and displacement amplitude show trends similar to the 


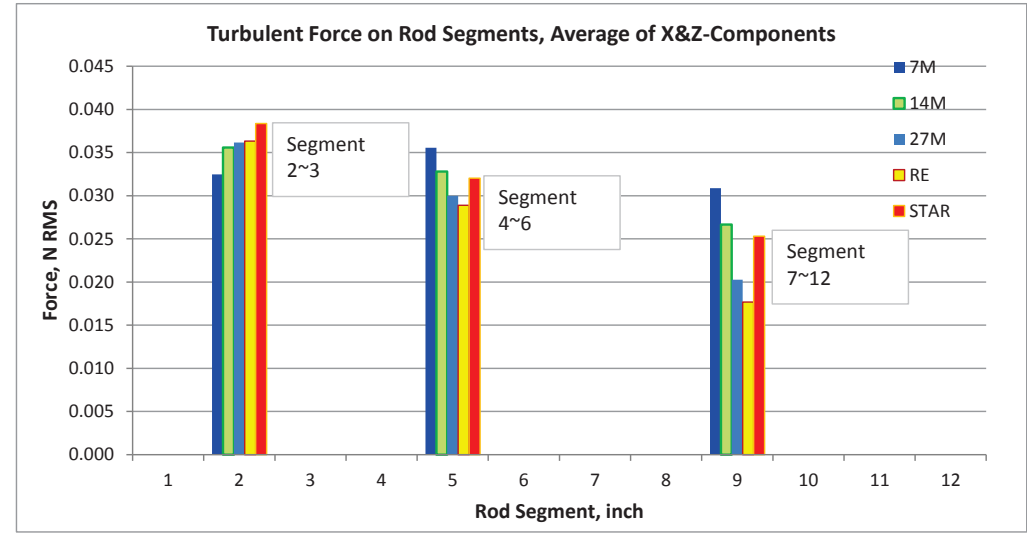

Figure 18: Lumped segment forces, average of $\mathrm{X}$ and $\mathrm{Z}$ components.

force distribution with the 48M CCM+ results matching most closely to the 14M Hydra-TH results on a span-by-span basis.

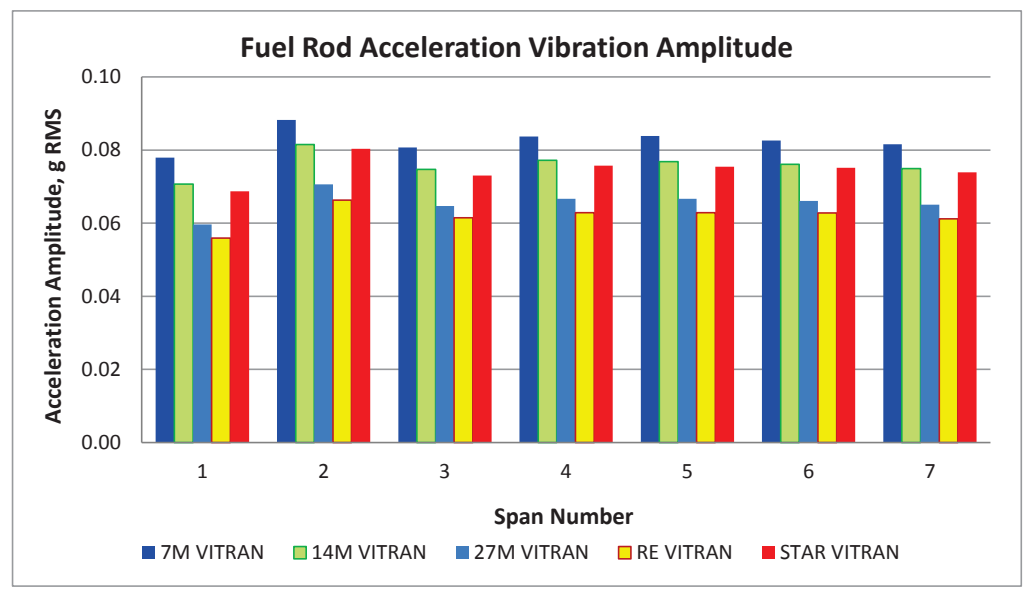

Figure 19: Acceleration distribution.

Figure 21 shows the computed wear work-rate at the 6 spacer grids (see Figure 4). Similar to the force distribution, the work-rate computed from the Hydra-TH results show convergent behavior relative to the mesh refinement used in the ILES computations. In addition, the work-rate based on the forces computed by Richardson extrapolation can be viewed as the limit of infinite spatial resolution for the ILES. On comparing work-rates obtained using Hydra-TH and STAR-CCM+, a close correspondence is seen between the 14M Hydra-TH and the 48M STAR-CCM+ values; they are seen to differ 


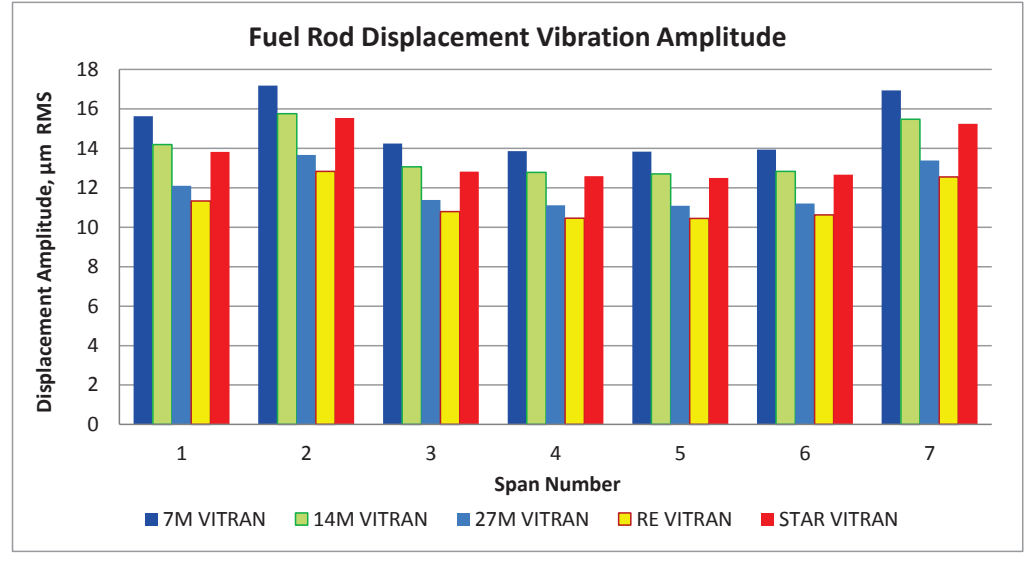

Figure 20: Displacement distribution.

by less than $2 \%$ across all of the spacer grids. Thus, compared to the $27 \mathrm{M}$ Hydra-TH-based and Richardson extrapolation-based work-rates, the $48 \mathrm{M}$ $\mathrm{CCM}+$ work-rate seems high.

The difference in the wear work-rate, computed relative to the work-rate computed using the RMS forces using Richardson extrapolation, is shown in Table 1. Here, we see a reduction from an average difference of $56.92 \%$ to $9.24 \%$ in going from the $7 \mathrm{M}$ mesh to the $27 \mathrm{M}$ mesh with Hydra-TH indicating that the Hydra-TH forces are converging under mesh refinement. In contrast, the differences for the 14M Hydra-TH results and the 48M STAR-CCM+ results are very similar despite the fact that the STAR-CCM+ results used $\approx 3.4 \times$ more mesh resolution. Although there is not definite evidence (e.g., a mesh-refinement study is lacking), it is likely that the difference derives from the use of wall functions in the STAR-CCM+ LES computations along with a relaxed $y^{+}$in the boundary layer regions. Despite these differences, the VITRAN results show that the CFD modeling methodologies used by HydraTH and STAR-CCM+, while different, produce comparable RMS forces, and ultimately, comparable acceleration, displacement and wear work-rates from VITRAN.

\section{Conclusions}

This paper presents a new approach for predicting GTRF induced fuel rod wear that uses ILES and nonlinear transient dynamics computations. The GTRF fluid-structure problem is separated into the simulation of the 


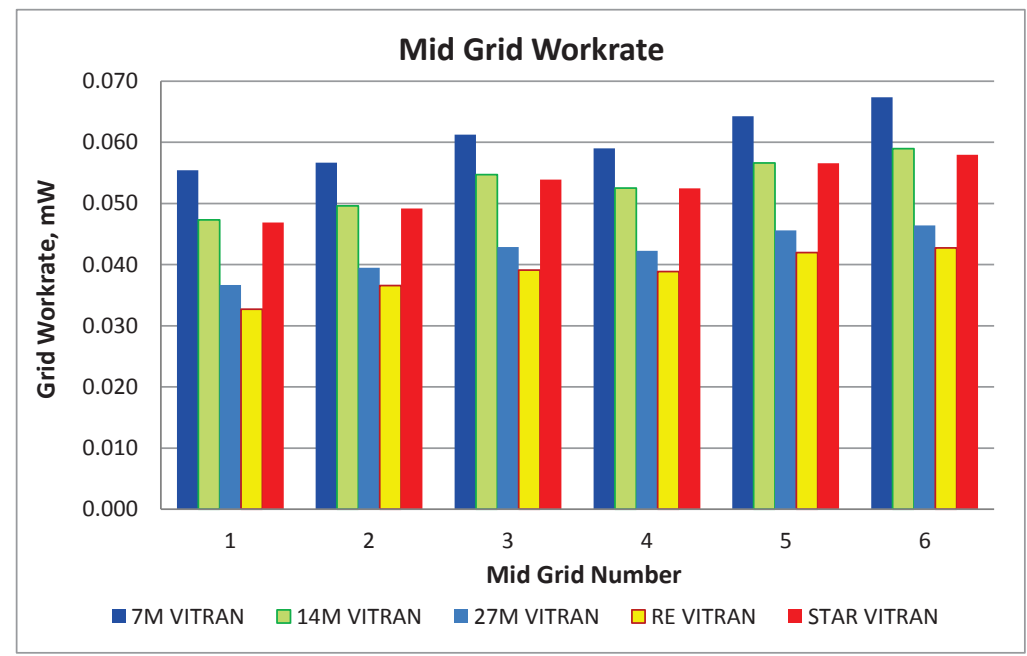

Figure 21: Wear work-rate.

turbulent flow field in the complex-geometry fuel-rod bundles, calculation of the statistics of the resulting fluctuating structural forces, and nonlinear transient dynamics analysis of the fuel rod. Ultimately, we envisage this approach to be used to improve reactor core designs so that fuel rod failure is minimized or potentially eliminated. As such, validation of the overall analysis procedure is of central importance. However, a prerequisite to performing such an overall validation study is a validation of the constituent subsystems individually.

This work represents progress largely towards validating the CFD-based thermal hydraulics capability for GTRF application. This progress was made by a) considering results from two different CFD solution algorithms in the the use of Hydra-TH and STAR-CCM+, b) considering and evaluating multiple turbulence-modeling frameworks - ILES, DES, and unsteady RANS (The Spalart-Allmaras RANS model tested was found to be inadequate for GTRF.), c) validating the ILES approach in a representative setting, d) considering and establishing mesh convergence for the series of ILES that were used to provide inputs to the structural dynamics computations, and by e) establishing uncertainty in the estimate of the structural forces due to turbulent intermittency.

One of the main findings of this work is that the fuel rod displacement, acceleration and wear work-rates as obtained from the nonlinear dynamics computations using VITRAN are relatively insensitive to the use of either 


\begin{tabular}{c|c|c|c|c}
$\begin{array}{c}\text { Span } \\
\text { Number }\end{array}$ & $7 \mathrm{M}$ & $14 \mathrm{M}$ & 27M & STAR-CCM+ \\
\hline 1 & $69.40 \%$ & $44.58 \%$ & $12.02 \%$ & $43.36 \%$ \\
2 & $54.92 \%$ & $35.66 \%$ & $8.02 \%$ & $34.41 \%$ \\
3 & $56.71 \%$ & $40.01 \%$ & $9.74 \%$ & $37.94 \%$ \\
4 & $51.83 \%$ & $35.19 \%$ & $8.81 \%$ & $35.02 \%$ \\
5 & $53.06 \%$ & $34.87 \%$ & $8.61 \%$ & $34.08 \%$ \\
6 & $57.69 \%$ & $38.02 \%$ & $8.71 \%$ & $35.70 \%$ \\
Average & $56.92 \%$ & $37.86 \%$ & $9.24 \%$ & $36.68 \%$
\end{tabular}

Table 1: Comparison of wear work-rate relative to the work-rate based on VITRAN calculations using the RMS forces from Richardson extrapolation

Hydra-TH or STAR-CCM+ for computing fluid forces on the rod. There was less than a $2 \%$ difference in the wear work-rate between a case that used forces from an intermediate resolution (14M) Hydra-TH computation and another that used forces from a high resolution (48M) STAR-CCM+ computation. Further analysis of the turbulent simulations using the two different methods leads us to hypothesize that the closer correspondence of the high-resolution STAR-CCM+ simulations to the intermediate resolution Hydra-TH simulation is related to the use of turbulent wall functions and a somewhat relaxed $y^{+}$consideration in meshing the boundary layer regions in STAR-CCM+. The use of such wall functions prevents further gains to be made beyond a certain resolution when the wall functions begin to dominate the solution.

Given the results of this study, a number of future directions can be identified to help validate the new approach to GTRF-related fuel rod wear prediction that we have presented. For example, besides further work on validation of the fuel rod vibration analysis itself, on the CFD side, a) Uncertainty in the statistics of structural forces due to alternative LES closures can be established by conducting additional studies with Hydra-TH that use the alternative LES closures. b) In order to assess the influence of channel cross-flow on the driving RMS forces for GTRF, a larger $5 \times 5$ tube bundle could be used to compute the forces on a central rod permitting direct simulation of the cross-flow effects. 


\section{Acknowledgments}

Los Alamos Report LA-UR-6-23692. This research was supported by the Consortium for Advanced Simulation of Light Water Reactors (http: //www . casl.gov), an Energy Innovation Hub (http://www.energy.gov/hubs) for Modeling and Simulation of Nuclear Reactors under U.S. Department of Energy Contract No. DE-AC05-00OR22725. This research used resources provided by the Los Alamos National Laboratory Institutional Computing Program, which is supported by the U.S. Department of Energy National Nuclear Security Administration under Contract No. DE-AC52-06NA25396. The authors gratefully acknowledge the experimental data for the $5 \times 5 \mathrm{rod}$ bundle provided by Elvis Dominguez-Ontiveros and Yassin Hassan at Texas A\&M.

\section{References}

[1] K. Liu, Heat transfer enhancement by three-dimensional surface roughness technique in nuclear fuel rod bundles, Master's thesis, Collge of Engineering and Computing, University of South Carolina (2014).

[2] M. P. Païdousis, A review of flow-induced vibrations in reactors and reactor components, Nuclear Engineering and Design 74 (1982) 31-60.

[3] M. J. Pettigrew, L. N. Carlucci, C. E. Taylor, N. J. Fisher, Flow-induced vibration and related technologies in nuclear technologies, Nuclear Engineering and Design 131 (1991) 81-100.

[4] T. Ikeno, T. Kajishima, Decay of swirling turbulent flow in rod-bundle, Journal of Fluid Science and Technology 1 (2006) 36-47.

[5] S. Benhamadouche, P. Moussou, C. L. Maitre, CFD estimation of the flow-induced vibrations of a fuel rod downstream of a mixing grid, in: PVP 2009 ASME Pressure Vessels and Piping 2009/Creep 8 Conference, Prague, Czech Republic, 2009.

[6] K.-T. Kim, The study on grid-to-rod fretting wear models for PWR fuel, Nuclear Engineering and Design 239 (2009) 2820-2824.

[7] K.-T. Kim, A study on the grid-to-rod fretting wear-induced fuel failure observed in the $16 \times 16 \mathrm{KOFA}$ fuel, Nuclear Engineering and Design 240 (2010) 756-762. 
[8] K.-T. Kim, The effect of fuel rod supporting conditions on fuel rod vibration characteristics and grid-to-rod fretting wear, Nuclear Engineering and Design 240 (2010) 1886-1391.

[9] M. E. Conner, E. Baglietto, A. M. Elmahdi, CFD methodology and validation for single-phase flow in PWR fuel assemblies, Nuclear Engineering and Design 240 (2010) 2088-2095.

[10] J. Yan, K. Yuan, E. Tatli, Z. Karoutas, A new method to predict gridto-rod fretting in a PWR fuel assembly inlet region, Nuclear Engineering and Design 241 (2011) 2974-2982.

[11] X. Zhang, S. D. Yu, Large eddy simulation of turbulent flow surrounding two simulated CANDU fuel bundles, Nuclear Engineering and Design 241 (2011) 3553-3572.

[12] A. Bhattachary, S. D. Yu, G. Kawall, Numerical simulation of turbulent flow through a 37 element CANDU fuel bundle, Annals of Nuclear Energy 40 (2012) 87-105.

[13] S. Delafontain, G. Ricciardi, Fluctuating pressure calculation induced by axial flow through mixing grid, Nuclear Engineering and Design 242 (2012) 233-246.

[14] Z. G. Lui, Y. Liu, J. Lu, Numerical simulation of the fluid-structure interaction for two simple fuel assemblies, Nuclear Engineering and Design 258 (2013) 1-12.

[15] A. Mohany, M. Hassan, Modeling of fuel bundle vibration and the associated fretting wear in a CANDU fuel channel, Nuclear Engineering and Design 264 (2013) $214-222$.

[16] P. M. Gresho, R. L. Sani, Incompressible flow and the finite element method, Advection-diffusion and isothermal laminar flow, John Wiley \& Sons, Chicester, England, 1998.

[17] F. F. Grinstein, L. G. Margolin, W. J. Rider, Implicit Large Eddy Simulation, Computing Turbulent Fluid Dynamics, Cambridge University Press, 2007. 
[18] M. A. Christon, J. Bakosi, B. T. Nadiga, M. Berndt, M. M. Francois, A. K. Stagg, Y. Xia, H. Luo, A hybrid incremental projection method for thermal-hydraulics applications, Journal of Computational Physics 317 (2016) $382-404$.

[19] S. B. Pope, Turbulent flows, Cambridge University Press, Cambridge, 2000.

[20] R. Y. Lu, Z. Karoutas, M. A. Christon, J. Bakosi, L. Pritchett-Sheats, CFD turbulence force calculations and grid-to-rod fretting simulation, Tech. Rep. CASL-I-2012-0165-000, Consortium for Advanced SImulation of Light-Water Reactors (CASL) (December 2012).

[21] M. A. Christon, Hydra-TH Theory Manual, Tech. Rep. LA-UR 1105387, Los Alamos National Laboratory (September 2011).

[22] J. Bakosi, M. Christon, R. B. Lowrie, L. Pritchett-Sheats, R. R. Nourgaliev, Large-eddy simulations of turbulent flow for grid-to-rod fretting in nuclear reactors, Nuclear Engineering and Design 262 (2013) 544-561.

[23] P. R. Rubiolo, R. Pomirleanu, R. Lu, D. Clayton, P. F. Joffre, D. V. D. V. P. Y. Young, An integrated fretting wear method: Development, features and experimental benchmark, in: Water Reactor Peformance Meeting, Top Fuel 2004, Orlando, Florida, USA, 2004.

[24] R. Y. Lu, Z. Karoutas, T. L. Sham, CASL virtual reactor predictive simulation: Grid-to-rod fretting wear, JOM 63 (8) (2011) 53 - 58.

[25] D. V. Paramov, M. Y. Young, S. J. King, Understanding fretting wear progression, in: International Meeting on LWR Fuel Performance, Germany, 2003.

[26] M. E. Conner, E. E. Dominguea-Otiveros, Y. A. Hassan, Hydraulic benchmark data for PWR mixing vane grid, in: The 14th International Topical Meeting on Nuclear Reactor Thermal Hydraulics, Toronto, Canada, 2011.

[27] J. Yan, M. E. Conner, R. A. Brewster, Z. E. Karoutas, E. E. DominguezOntiveros, Y. A. Hassan, Validation of CFD method in predicting steady and transient flow field generated by PWR mixing vane grid, in: CFD4NRS-4 - The Experimental Validation and Application of CFD 
and CMFD Codes in Nuclear Reactor Technology, OECD/NEA \& International Atomic Energy Agency (IAEA) Workshop, Daejeon, Korea, 2012.

[28] J. Yan, personal communication (August 2012).

[29] A. M. Elmahdi, R. Lu, M. E. Conner, Z. Karoutas, E. Baglietto, Flow induced vibration forces on a fuel rod by LES CFD analysis, in: The 14th International Topical Meeting on Nuclear Reactor Thermal Hydraulics (NURETH-14), Hilton Toronto Hotel, Toronto, Ontario, Canada, 2011.

[30] L. F. Richardson, J. A. Gaunt, The deferred approach to the limit. part i. single lattice. part ii. interpenetrating lattices, Philosophical Transactions of the Royal Society of London. Series A, containing papers of a mathematical or physical character (1927) 299-361. 